\title{
Mixture-Based Path Clustering for Synthesis of ECMWF Ensemble Forecasts of Tropical Cyclone Evolution ${ }^{\mathscr{O}}$
}

\author{
PrabHani Kuruppumullage Don \\ Department of Biostatistics and Computational Biology, Dana-Farber Cancer Institute, and \\ Department of Biostatistics, Harvard T.H. Chan School of Public Health, Boston, Massachusetts \\ JENNI L. EVANS \\ Department of Meteorology, The Pennsylvania State University, University Park, Pennsylvania \\ FRANCESCA CHIAROMONTE \\ Department of Statistics, The Pennsylvania State University, University Park, Pennsylvania \\ Alex M. KowALESKI \\ Department of Meteorology, The Pennsylvania State University, University Park, Pennsylvania
}

(Manuscript received 8 June 2015, in final form 12 May 2016)

\begin{abstract}
In this article, three tropical cyclones and their 120-h, 50-member ECMWF Integrated Forecasting System (IFS) ensemble track forecasts at 10 initialization times are considered. The IFS forecast tracks are clustered with a regression mixture model, and two traditional diagnostics (the Bayesian information criterion and a measure of strength of cluster assignment) are used to determine the optimal polynomial order and number of clusters to use in the model. In addition, cross-validation versions of the two diagnostics are formulated and computed to further aid in model selection. Both traditional and cross-validation diagnostics suggest that third-order polynomials and five clusters are effective options-although the evidence is less conclusive for the number of clusters than for the polynomial order, and the cross-validation diagnostics favor a smaller number of clusters than the traditional ones.

Path clustering of IFS tropical cyclone track forecasts with this third-order polynomial, five-cluster regression mixture model produces interpretable partitions by direction and speed of motion for each of the storms and initialization times considered. Thus, this approach effectively synthesizes the forecast spreads within the IFS into a small number of representative trajectories. Based on how forecasts distribute across clusters, this approach also provides information on the likelihood of each such representative trajectory. If used operationally, this information has the potential to aid forecasters in parsing and quantifying the uncertainty in tropical cyclone track forecasts.
\end{abstract}

Supplemental information related to this paper is available at the Journals Online website: http://dx.doi.org/10.1175/ MWR-D-15-0214.s1.

Corresponding author address: Jenni L. Evans, Department of Meteorology, The Pennsylvania State University, 503 Walker Building, University Park, PA 16802.

E-mail: jle7@psu.edu

\section{Introduction}

While tropical cyclone (TC) track forecasts have improved substantially during the last 25 years (NHC 2015), track forecasts for certain cyclones continue to pose substantial challenges. This is especially true for TCs undergoing extratropical transition (ET), for which small errors in track (or structure) forecasts can substantially impact forecasts of the evolving storm (e.g., 
Evans et al. 2006; Veren et al. 2009), as well as forecasts of downstream synoptic-scale disturbances due to changes in the planetary wave pattern (Jones et al. 2003; Harr et al. 2008; Harr and Dea 2009; Keller et al. 2014).

Ensemble prediction systems (EPSs) provide a method of quantifying the uncertainty in numerical model forecasts. In this study we focus on TC track forecasts produced by a global EPS. Through variations in initial conditions and/or model physics, an EPS can capture the spread of potential tracks and storm structures associated with a TC. It can also provide an assessment of likelihood by showing which storm tracks and structural evolutions occur more frequently within the EPS.

One challenge for effectively using EPS forecasts is in the choice of "metric" used to capture the spread and distribution of forecast outcomes. Condensing the EPS forecasts into a single ensemble mean can result in a meaningless forecast: for example, the mean of an EPS forecast of a bifurcation in forecast TC tracks will be a track that no member predicts. In contrast, clustering provides an objective method of grouping ensemble members with related features by requiring maximum within-cluster similarity and among-cluster differences.

Clustering (primarily $k$-means point clustering) has already been used to analyze TC structural evolution during ET, as well as ensemble forecasts of the large-scale synoptic pattern during and after ET (Arnott et al. 2004; Evans et al. 2006; Harr et al. 2008; Veren et al. 2009; Keller et al. 2011, 2014). Here we apply and evaluate a statistical method for path clustering of ensemble track forecasts based on regression mixture models (Gaffney et al. 2007). Path clustering offers an obvious advantage relative to point clustering; namely, the ability to exploit the spatiotemporal nature of the data. Mixture models also offer advantages, chief among them the probabilistic treatment of cluster assignments, and have been used successfully in partitioning climatological TC tracks (Camargo et al. 2007; Kozar et al. 2012; Paliwal and Patwardhan 2013).

Importantly, employing regression mixture models for path clustering of EPS track forecasts requires choosing model specifications. For many datasets, this is a difficult problem - and if the data are complex, the choice is often not straightforward. We tackle this problem by considering several model selection diagnostics at once. In particular, we use two traditional and well-understood diagnostics, but also formulate and compute cross-validation versions of both.

In this study, we consider the 50 ensemble forecasts from the Integrated Forecasting System (IFS) of the

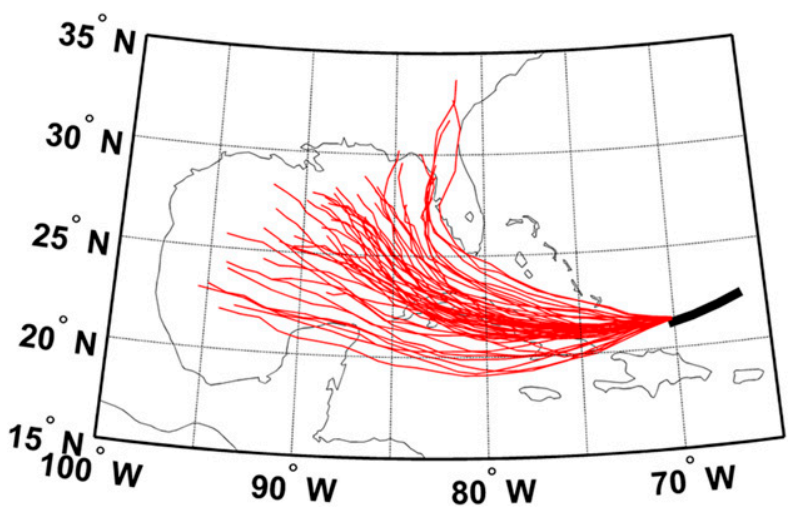

FIG. 1. The 50 ECMWF IFS 120-h forecast tracks for Hurricane Ike initialized at 0000 UTC 7 Sep 2008. Tracks range from landfall on the Yucatan Peninsula to landfall on the Florida Peninsula.

European Centre for Medium-Range Weather Forecasts (ECMWF; Buizza 2006; Fig. 1) and cluster 120-h track forecasts for a total set of 30 initialization times drawn from three TCs: western North Pacific Typhoons Sinlaku (2008) and Noul (2015), and North Atlantic Hurricane Ike (2008). To set the stage, we review the synoptic history of the three TCs (section 2). Next, we provide general descriptions of the ECMWF IFS (section 3) and of path clustering with regression mixture models as applied to IFS track forecasts (sections 4 and 5). Diagnostics used for model selection are described in section 6 , our clustering results are presented in section 7 , and a summary discussion with conclusions is provided in section 8 .

\section{Tropical cyclone synoptic histories}

Three contrasting TCs are used in this study to illustrate path clustering with regression mixture models and its application to EPS forecasts. The evolution of each of these systems is summarized here, with key times highlighted in Tables 1-3.

Typhoon Sinlaku developed from a tropical depression that formed in the Philippine Sea on 7 September 2008. The storm reached its maximum intensity of $125 \mathrm{kt}$ $\left(64 \mathrm{~m} \mathrm{~s}^{-1}\right.$; equivalent to category 4 on the Saffir-Simpson hurricane wind scale on 10 September, making landfall in northern Taiwan on 13 September as a 100-kt $\left(51 \mathrm{~m} \mathrm{~s}^{-1}\right)$ typhoon. Sinlaku weakened over Taiwan, and further weakened into a minimal tropical storm as it drifted eastnortheastward over the East China Sea in a region of strong vertical wind shear. On 17 September, Sinlaku began to accelerate to the east-northeast and reintensify, regaining typhoon intensity while just off the east coast of Japan. As Sinlaku interacted with a midlatitude trough, it underwent ET and was declared 
TABLE 1. Initialization times used for clustering IFS forecasts of Typhoon Sinlaku (2008) along with storm intensity at those times and storm life cycle milestones.

\begin{tabular}{rccc}
\hline \hline ID No. & Initial time & Max sustained winds $\left(\mathrm{m} \mathrm{s}^{-1} ;\right.$ JTWC $)$ & Storm milestone \\
\hline 1 & 0000 UTC 14 Sep & 46 & Just after landfall in Taiwan \\
2 & 1200 UTC 14 Sep & 33 & Emerges over East China Sea \\
3 & 0000 UTC 15 Sep & 31 & - \\
4 & 1200 UTC 15 Sep & 31 & Strong vertical wind shear causes weakening \\
5 & 0000 UTC 16 Sep & 23 & Slowly drifting eastward \\
6 & 1200 UTC 16 Sep & 21 & Begins to move east-northeastward \\
7 & 0000 UTC 17 Sep & 18 & Begins to reintensify \\
8 & 1200 UTC 17 Sep & 23 & Near southern coast of Japan \\
9 & 0000 UTC 18 Sep & 31 & 33 \\
10 & 0000 UTC 19 Sep & &
\end{tabular}

extratropical by the JTWC at 1200 UTC 20 September (JTWC 2015; Table 1; Fig. 2a).

Typhoon Noul originated from a tropical depression that formed southwest of Chuuk, Micronesia, on 2 May 2015. As Noul drifted slowly to the northwest, then west-southwest, it intensified, becoming a typhoon early on 6 May. Typhoon Noul continued to intensify as it accelerated west-northwestward toward the Philippines. It underwent rapid intensification on 8 and 9 May, reaching its maximum intensity of $145 \mathrm{kt}$ ( $75 \mathrm{~m} \mathrm{~s}^{-1}$; category 5) early on 10 May, then passed just offshore of the northern tip of Luzon later that day. Noul began to weaken and recurved to the northwest and north in an environment of strong vertical wind shear. Noul weakened to tropical storm intensity on 12 May and was declared extratropical later that day (JTWC 2015; Table 2; Fig. 2a).

Ike was located in the central tropical Atlantic when it was classified as a tropical storm on 1 September 2008. It moved to the west-northwest, steadily intensifying to hurricane strength two days later, then intensifying rapidly until reaching its maximum intensity of $125 \mathrm{kt}\left(64 \mathrm{~m} \mathrm{~s}^{-1}\right.$; category 4$)$ on 4 September. Ike slowly weakened over the next two days due to increased vertical wind shear. The shear abated on
6 September and Ike reintensified; it traversed Turks and Caicos before making landfall in eastern Cuba on 8 September. Ike emerged into the western Caribbean Sea as a category 1 hurricane, crossing western Cuba and emerging into the Gulf of Mexico. Ike slowly intensified as it moved toward the Texas coast. It made landfall on Galveston Island early on 13 September with maximum sustained winds of $95 \mathrm{kt}\left(49 \mathrm{~m} \mathrm{~s}^{-1}\right)$. Ike weakened and was classified extratropical at 1200 UTC 14 September while over southern Missouri. The extratropical low moved northeastward over the Ohio Valley as a major rain and wind event, before weakening and moving into Ontario, Canada (Berg 2009; Table 3; Fig. 2b).

\section{The ECMWF Integrated Forecasting System}

Operational ECMWF IFS forecasts are the basis of this study. The configuration of the operational ECMWF IFS varied between 2008 and 2015: in 2008 it had an approximately $80-\mathrm{km}$ horizontal resolution (TL255) and 40 vertical levels (Buizza et al. 2003); in 2015 the IFS resolution had increased to $32 \mathrm{~km}$ (T639) and 91 vertical levels (ECMWF 2015). Diabatic tropical singular vectors are computed in target areas centered

TABLE 2. Initialization times used for clustering IFS forecasts of Typhoon Noul (2015) along with storm intensity at those times and storm life cycle milestones.

\begin{tabular}{cccc}
\hline \hline ID No. & Initial time & Max sustained winds $\left(\mathrm{m} \mathrm{s}^{-1} ;\right.$ JTWC $)$ & Storm milestone \\
\hline 1 & 0000 UTC 4 May & 18 & Drifting slowly west-northwestward \\
2 & 1200 UTC 4 May & 18 & - \\
3 & 0000 UTC 5 May & 28 & Drifting slowly westward \\
4 & 1200 UTC 5 May & 33 & Intensifies to typhoon \\
5 & 0000 UTC 6 May & 33 & - \\
6 & 1200 UTC 6 May & 41 & Begins to accelerate west-northwestward \\
7 & 0000 UTC 7 May & 46 & - \\
8 & 1200 UTC 7 May & 51 & Intensifies to category-3-equivalent typhoon \\
9 & 0000 UTC 8 May & 51 & - \\
10 & 1200 UTC 8 May & 54 & - \\
\hline
\end{tabular}


TABLE 3. Initialization times used for clustering IFS forecasts of Hurricane Ike (2008) along with storm intensity at those times and storm life cycle milestones.

\begin{tabular}{rccl}
\hline \hline ID No. & Initial time & Max sustained winds $\left(\mathrm{m} \mathrm{s}^{-1} ;\right.$ NHC) & Storm milestone \\
\hline 1 & 0000 UTC 4 Sep & 54 & Rapidly intensifies to major hurricane \\
2 & 1200 UTC 4 Sep & 62 & Just after maximum intensity \\
3 & 0000 UTC 5 Sep & 59 & Begins to move west-southwestward due to strong \\
4 & 1200 UTC 5 Sep & 54 & ridge to north \\
& & 51 & Weakens to category-2 hurricane due to northerly shear \\
5 & 0000 UTC 6 Sep & 49 & Reintensifies to category 4 \\
6 & 1200 UTC 6 Sep & 59 & Landfall in eastern Cuba \\
7 & 0000 UTC 7 Sep & 57 & Over eastern Cuba \\
8 & 1200 UTC 7 Sep & 59 &
\end{tabular}

on a TC of at least tropical-storm strength (Barkmeijer et al. 2001; Puri et al. 2001). These singular vector initial perturbations are designed to produce optimal growth over a 48-h time period, maximizing the ensemble spread at this forecast time (Leutbecher and Palmer 2008). Along with the tropical singular vectors, 25 extratropical singular vectors in each hemisphere are used to generate initial conditions for the 50 perturbed forecasts; the ensemble control model is the 51st IFS member (Buizza et al. 2003). In addition to singular vector-derived initial perturbations, the ECMWF IFS uses stochastic physics (randomly perturbing the model parameterizations) to account for inherent model error due to parameterizing unresolved physical processes. Forecast storm positions from each IFS ensemble member are produced every $6 \mathrm{~h}$ from the initialization time out to $120 \mathrm{~h}$ ( 5 days); however, in some cases the forecast track for some (or possibly all) IFS members terminates before $120 \mathrm{~h}$.

In this study, we cluster IFS forecasts from 10 initialization times for each of the three TCs under consideration using a regression mixture model (see below). For Sinlaku, initialization times are subsequent to the time of maximum intensity, including the period during which it began to undergo ET south of Japan (Table 1). For Noul, initialization times are from the period when it was in the deep tropics, before it reached maximum intensity (Table 2). For Ike, initialization times are from the period when it was a mature, intense hurricane (Table 3). Thus, the data we use comprise a variety of states within the life cycle of TCs.

\section{An overview of path clustering with regression mixture models}

We use path clustering with regression mixture models (Gaffney et al. 2007) for our analysis of IFS track forecasts. There are two key reasons for this choice: 1) path clustering incorporates information over the entire forecast period, exploiting the spatiotemporal nature of the data (tracks are described over time in 2D space; partitioning the points they comprise, as opposed to the full tracks, inevitably leads to a loss of information); and 2) mixture models treat cluster assignments probabilistically. By fitting appropriate regression mixture models,
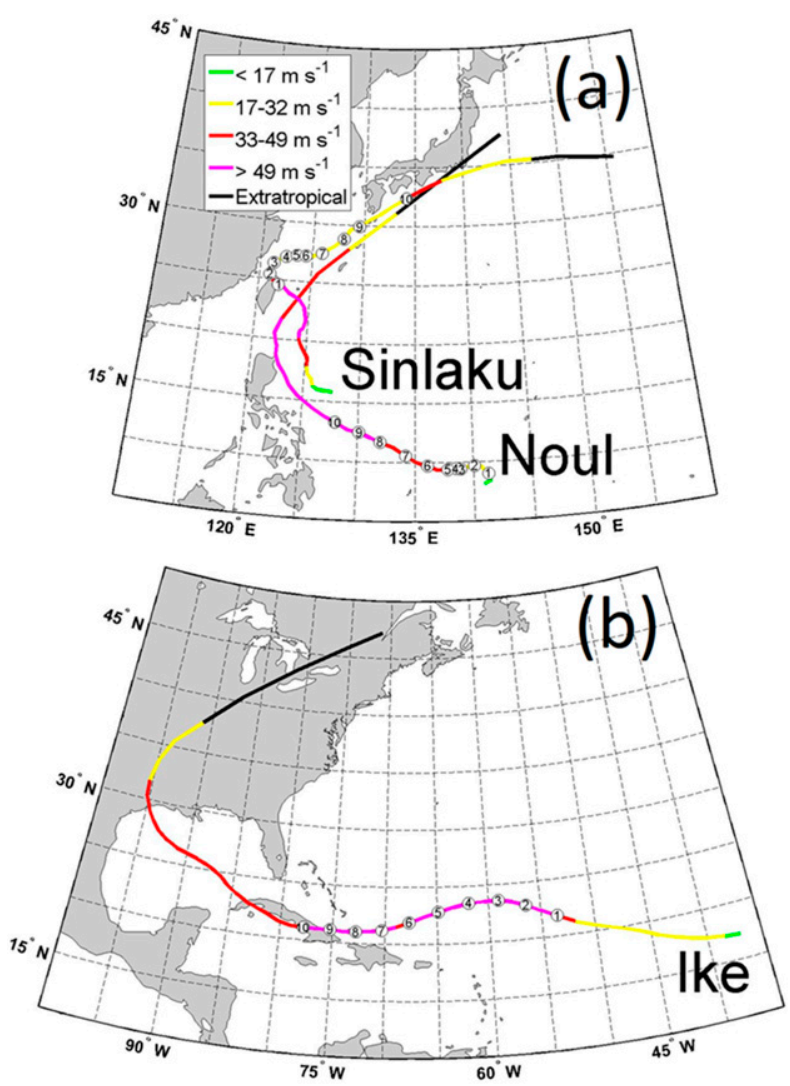

FIG. 2. Observed tracks of (a) Typhoons Sinlaku (2008) and Noul (2015) and (b) Hurricane Ike (2008). Numbered circles indicate the positions for each storm at the 10 initialization times of ECMWF IFS forecasts used in clustering and reported in Tables 1-3 and 5-7. 
TABLE 4. Features of the regression mixture model used for path clustering.

\begin{tabular}{lcc}
\hline \multicolumn{1}{c}{ Feature } & \multicolumn{1}{c}{ Purpose } & Specifications tested \\
\hline No. of clusters & $\begin{array}{c}\text { Select a number of clusters appropriate to capture the } \\
\text { spread of the data } \\
\text { Represent the central (mean) trajectory of each cluster } \\
\text { as a polynomial, and select the polynomial order } \\
\text { Represent the cross section of each cluster as elliptical, } \\
\text { and select its volume, eccentricity, and orientation }\end{array}$ & First- through fifth-order polynomials \\
Geometry of clusters & Constant elliptical cross sections (fixed)
\end{tabular}

we are able to partition 120 -h forecast tracks into a small number of clusters, with confidence in membership assignments expressed as probabilities. Geometrically, the clusters used are probabilistic "flexible pipes" unfolding over time in the $2 \mathrm{D}$ space described by latitude and longitude. As shown in section 6 , these pipes can generally be well described as centered about low-order polynomials.

In full generality, when mixture models are used for clustering, observations are regarded as draws from a mixture of component probability distributions (typically Gaussians), each of which corresponds to a cluster. Posterior or "membership" probabilities, which measure the congruency of each observation to each component, are used to produce cluster assignments. Probabilistic assignments provide more information than a hard partition, in which each observation is deterministically assigned to one cluster only, because they indicate the assignment strength of individual observations to each cluster. Furthermore, they provide an evaluation criterion: a superior cluster solution has a larger fraction of observations with strong cluster assignment (maximum posterior probability near 1).

In our application, observations are forecast TC tracks and are regarded as draws from a mixture of $K$ components. Each component is a Gaussian about a polynomial trajectory in 2D and corresponds to a cluster (i.e., a "pipe"). Thus, we have $n$ tracks $\mathbf{Y}=\left\{\mathbf{y}_{1}, \mathbf{y}_{2}, \ldots\right.$, $\left.\mathbf{y}_{n}\right\}$, with $\mathbf{y}_{i}$ comprising $n_{i}$ points in $\mathbb{R}^{2}$ at corresponding times $t_{i}$. The relationship between $\mathbf{y}_{i}$ and $t_{i}$ is modeled as a component-specific $q$ th-order polynomial with an additive Gaussian error:

$$
\mathbf{y}_{i}=\mathbf{T}_{i} \boldsymbol{\beta}_{k}+\boldsymbol{\epsilon}_{i} \quad \boldsymbol{\epsilon}_{i} \sim N\left(0, \mathbf{\Sigma}_{k}\right),
$$

where $\boldsymbol{\beta}_{k}$ and $\boldsymbol{\Sigma}_{k}$ are, respectively, regression coefficients and error covariance for the $k$ th component, and $\mathbf{T}_{i}$ is

$$
\mathbf{T}_{i}=\left[\begin{array}{cc}
\mathbf{X}_{i} & 0 \\
0 & \mathbf{X}_{i}
\end{array}\right] \text { where } \quad \mathbf{X}_{i}=\left[\begin{array}{ccccc}
1 & t_{i 1} & t_{i 1}^{2} & \cdots & t_{i 1}^{q} \\
\vdots & \vdots & \vdots & \cdots & \vdots \\
1 & t_{i n i} & t_{i n i}^{2} & \cdots & t_{i n i}^{q}
\end{array}\right]
$$

The density of the forecast tracks $\mathbf{Y}$ is thus

$$
p(\mathbf{y} \mid \mathbf{T}, \boldsymbol{\Theta})=\prod_{i=1}^{n} \sum_{k=1}^{K} \tau_{k} N\left(\mathbf{y}_{i} \mid \mathbf{T}_{i} \boldsymbol{\beta}_{k}, \mathbf{\Sigma}_{k}\right),
$$

where $\tau_{k}$ is the probability of the $k$ th component $\left(\tau_{k} \geq 0\right.$, $\left.\Sigma \tau_{k}=1\right)$ and $\boldsymbol{\Theta}$ comprises all $\tau_{k}, \boldsymbol{\beta}_{k}$, and $\boldsymbol{\Sigma}_{k}$ parameters (see the appendix for details). Tracks are assigned to pipes (clusters) probabilistically using the maximum posterior probability rule:

$$
\mathbf{y}_{i} \in \text { cluster } k \quad \text { if } \quad \omega_{i k}=\underset{g}{\operatorname{argmax}} \omega_{i g},
$$

where $\omega_{i k}$ is the posterior probability of the $i$ th track belonging to the $k$ th component.

\section{a. Parameterization of a mixture model}

When mixture models are used for clustering, one must specify the number of clusters $(K)$ appropriate to capture the complexity of the data, as well as the geometric characteristics of each cluster (Table 4); the geometric characteristics are expressed through model parameters that are then estimated on the data (see the appendix for details). In simple point clustering (McLachlan and Basford 1987; Fraley and Raftery 2009) these parameters represent (i) cluster centers and (ii) cluster geometryvolume, eccentricity, and orientation of the clusters (i.e., the Gaussian components' mean vectors and covariance matrices). Prior to estimation, these parameters may be subject to constraints (e.g., one can require that all clusters are spherical, or have equal volume).

In regression mixture-model path clustering, (i) cluster centers become trajectories in the space of interest (here latitude and longitude), with shapes parameterized as polynomials and constrained in terms of their order. The volume, eccentricity, and orientation parameters in (ii) define variation about these trajectories (Table 4).

\section{b. Estimation of mixture model parameters via the expectation-maximization algorithm}

Once a mixture model is specified, its parameters and the posterior probabilities of each observation belonging to each cluster are estimated using the 
expectation-maximization (EM) algorithm (McLachlan and Basford 1987). The algorithm maximizes the likelihood function iteratively, each iteration comprising two steps (see the appendix for details):

- The E step: New estimates of the posterior probabilities are computed, given the current estimates of the model parameters.

- The M step: New estimates of the model parameters are computed, given the current estimates of the posterior probabilities.

These steps are repeated until convergence criteria are met, at which point unique estimates are available for all model parameters and posterior probabilities. For example, we may have parameter estimates of the coefficients of third-order polynomials representing the central trajectories of (say) five clusters and the volumes of their elliptical cross sections. Before the first iteration of EM, the number of clusters, order of the polynomials, and geometry of clusters (Table 4) must be prescribed. For the purpose of model selection, the EM is run multiple times on candidate models with varying specifications, and the candidates are evaluated using the diagnostics described now.

\section{Diagnostic methods for mixture model selection}

To obtain a clustering solution for a given storm and initialization time, we must prescribe the number of clusters, polynomial order, and cluster geometry to use in the mixture model. After evaluating a variety of options, we assumed a cluster geometry with constant elliptical cross sections along our pipes (i.e., clusters). Thus, we are left to provide objective guidance in selecting the number of clusters and polynomial order. We employ two traditional model selection diagnostics: the Bayesian information criterion (BIC) and a measure of the strength of cluster assignment $\left(F_{0.95}\right.$ : the percentage of observations with maximum posterior probabilities below 0.95 ). We complement these diagnostics by formulating a cross-validation version of each. This provides an additional perspective in selecting regression mixture model specifications.

\section{a. Traditional diagnostics: Bayesian information criterion and strength of cluster assignment}

The BIC is a penalized version of the maximum loglikelihood (MLL); that is, the value reached through the EM iterations when maximizing the log-likelihood function to estimate parameters for a given model specification. MLL always favors more complex models (larger number of clusters, higher polynomial order).

The BIC is calculated from the MLL, with a penalty based on the number of independent parameters to be estimated and the number of observations. In full generality, let $m$ be a candidate model specification comprising $p(m)$ independent parameters, and $L_{n}(m)$ be the MLL based on $n$ observations. Then the BIC for $m$ is given by $\operatorname{BIC}(m)=-2 L_{n}(m)+p(m) \ln (n)$ [note that smaller BIC values indicate greater support for a model specification; see Fraley and Raftery (2009)]. Because of the penalty, a more complex model will not always have a superior (smaller) BIC value compared to a less complex model. Thus, the BIC favors specifications that provide a balance between likelihood maximization and parsimony.

In parallel with the BIC, one can use the posterior probabilities estimated by the EM for each observation to create a different evaluation of a model specification. A maximum estimated posterior probability close to 1 means that the observation is unambiguously assigned to its "best fitting" cluster. Thus, one way to summarize the strength of cluster assignments is to calculate the fraction of observations with maximum estimated posterior probabilities below a certain threshold $F_{\eta}$; here we use the threshold $\eta=0.95$. In symbols, if $\omega_{i}^{*}$ denotes the maximum estimated posterior probability of observation $i$ and $I(\cdot)$ is the indicator function, $F_{0.95}(m)=$ $1 / n \sum_{i=1}^{n} I\left(\omega_{i}^{*}<0.95\right)$, so higher values of $F_{0.95}$ indicate that a larger fraction of cluster assignments are ambiguous. In other words, higher values of $F_{0.95}$ correspond to weaker support for a model specification.

Among a collection of candidate model specifications $M$, we seek to choose the $m \in M$ with the best qualitative combination of $\operatorname{BIC}(m)$ and $F_{0.95}(m)$ values, and adopt the cluster solution produced by estimating its parameters and posterior probabilities through the EM algorithm.

\section{b. Cross-validation diagnostics}

We formulate variants of BIC and $F_{0.95}$ using cross validation. These provide a complementary and potentially more robust perspective on model selection because, for each model specification, cross validation produces a set of values of the two diagnostics. This allows one to gauge the variability intrinsic in their assessment. We proceed as follows, For $j=1,2, \ldots, J$ (say $J=100)$ :

(i) Randomly split the observations to be clustered (i.e., the 50 IFS ensemble tracks) into a training set and a test set $\left(\mathrm{TR}_{j}\right.$ and $\left.\mathrm{TE}_{j}\right)$ with $S \%$ of the data in the former (say $S=80$, so 40 tracks are randomly assigned to the training set, and the remaining 10 to the test set).

(ii) For each model specification $m \in M$ (i.e., each combination of polynomial order and number of 
clusters), compute EM parameter estimates $\hat{\boldsymbol{\tau}}_{k}, \hat{\boldsymbol{\beta}}_{k}, \hat{\boldsymbol{\Sigma}}_{k}, k=1,2, \ldots, K$ based solely on observations in $\mathrm{TR}_{j}$.

(iii) Use parameter estimates from (ii) to compute a $\mathrm{BIC}$ value for $m$ on $\mathrm{TE}_{j}$ as

$$
\begin{aligned}
\operatorname{BIC}_{j}(m)= & -2 \sum_{i \in \mathrm{TE}_{j}} \log \sum_{k=1}^{K} \hat{\tau}_{k} N\left(\mathbf{y}_{i} \mid \mathbf{T}_{i} \hat{\boldsymbol{\beta}}_{k}, \hat{\mathbf{\Sigma}}_{k}\right) \\
& +p(m) \log \left(n_{\mathrm{TE}_{j}}\right)
\end{aligned}
$$

where $n_{\mathrm{TE}}$ indicates the number of ensemble tracks in the test set.

(iv) Use parameter estimates from (ii) to generate estimated posterior probabilities for each observation in $\mathrm{TE}_{j}$, take their maxima, and compute the fraction of weaker assignments as

$$
F_{0.95, j}(m)=\frac{1}{n_{\mathrm{TE}_{j}}} \sum_{i \in \mathrm{TE}_{j}} I\left(\omega_{i}^{*}<0.95\right) .
$$

For each candidate model specification $m \in M$, this process will produce sets of measurements $\mathrm{BIC}_{j}(m)$ and $F_{0.95, j}(m)$ over the cross-validation iterations $j=1,2, \ldots$, $J$. These measurements can be summarized by their means and standard deviations, which can then be employed, along with the traditional $\mathrm{BIC}(m)$ and $F_{0.95}(m)$ values, for selecting a model specification. Once the model is chosen, its parameters and posterior probabilities are estimated via EM on the entire (rather than training) dataset (50 ensemble tracks), and the corresponding cluster solution is adopted. In our application, the collection of candidate model specifications is given by all combinations of two-seven clusters and first-fifth polynomial order (cluster cross sections are always taken to be elliptical and constant along the pipes). Here $J$ is set to 100 and $S$ is set to 80 .

\section{Path clustering with regression mixture models applied to IFS track forecasts}

To illustrate this approach we describe the selection of a satisfactory model specification and the corresponding clustering solution for a single storm and forecast initialization time, then we examine the application of our approach across all initialization times under consideration.

\section{a. An example: Hurricane Ike from 0000 UTC 7 September 2008}

We consider the set of 50 IFS forecasts of Hurricane Ike initialized at 0000 UTC 7 September 2008 (Fig. 1). At this time Ike was a category 4 hurricane about to make landfall in eastern Cuba. In the subsequent five days, Ike would cross eastern Cuba and weaken to a category 1 hurricane, before moving into the Gulf of Mexico and reintensifying to a category 2 hurricane (Table 3). Tracks derived from the IFS members initialized at this time vary between making landfall on the Yucatan Peninsula of Mexico to landfall in Florida; however, no natural partition of these tracks is obvious from visual inspection (Fig. 1). These data provide an obvious challenge to the interested onlooker and a test for the clustering approach we propose.

\section{1) Model Selection using traditional DIAGNOSTICS}

The traditional diagnostics $\mathrm{BIC}(m)$ and $F_{0.95}(m)$ (section $5 \mathrm{a}$ ) for model specifications with polynomial trajectories of orders $1-5$, and between 2 and 7 clusters [based on the cyclone structure clustering results of Arnott et al. (2004) and Veren et al. (2009)] are depicted in Figs. 3a and 3c. Recall that for both diagnostics lower values indicate better model specifications.

The traditional BIC decreases greatly switching from first- to second-order polynomials, and still substantially switching from second to third order. No notable decrease in BIC is achieved by further increasing the polynomial order, indicating that cubic trajectories are sufficient for capturing the spatial patterns of IFS track forecasts at this initialization time. On the other hand, the traditional BIC does not give a clear indication for a satisfactory number of clusters; for every polynomial order, the BIC keeps decreasing as the number of clusters increases up to seven, although the rate of decrease appears to taper off after five clusters (Fig. 3a).

The traditional $F_{0.95}$ indicates that all model specifications produce strong probabilistic cluster assignments: for every polynomial order and number of clusters combination, at least $96 \%$ (48 of 50 IFS members) have maximum posterior probabilities of 0.95 or greater (Fig. 3c). Thus, this diagnostic provides little discrimination among model specifications. Notwithstanding the narrow range, third-order polynomials are parsimoniously the best performing, with $F_{0.95}=0$ from three clusters onward.

In summary, for this example traditional diagnostics support the choice of third-order polynomial trajectories, but do not clearly discriminate among different number of clusters.

\section{2) MOdEL SELECTION USING CROSS-VALIDATION DIAGNOSTICS}

Means and standard deviations of the diagnostics $\mathrm{BIC}_{j}(m)$ and $F_{0.95, j}(m), j=1,2, \ldots, 100$, generated by cross validation (section 5b) are plotted in Figs. 3b,d. Model parameter ranges tested are the same as for the traditional diagnostics. 

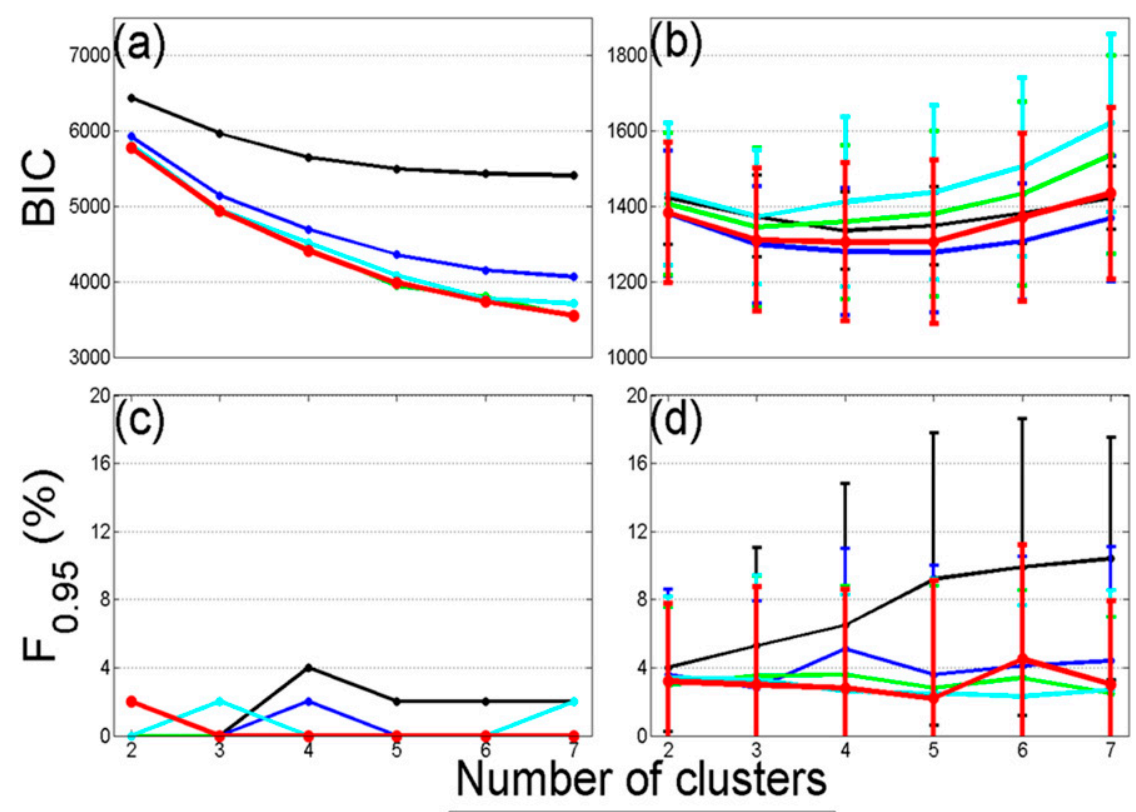

$$
\rightarrow 1^{\text {st }} \rightarrow 2^{\text {nd }} \rightarrow 3^{\text {rd }} \rightarrow 4^{\text {th }} \rightarrow 5^{\text {th }}
$$

FIG. 3. Evaluation of various regression mixture model specifications used in clustering Hurricane Ike ECMWF IFS forecasts initialized at 0000 UTC 7 Sep: (a) traditional BIC, (b) cross-validation BIC, (c) traditional $F_{0.95}$, and (d) cross-validation $F_{0.95}$. The order of polynomial trajectories is indicated by line color, and the number of clusters is on the horizontal axis. Vertical bars in (b) and (d) represent values that are \pm 1 standard deviation from the mean cross-validation BIC and $F_{0.95}$ values.

Interestingly, even though the broad standard deviations suggest caution in drawing conclusions, the cross-validation BIC suggests more parsimonious model specifications than the traditional BIC. Second-order polynomial trajectories perform as well (for small number of clusters) or better (for larger number of clusters) than third-order polynomials. Moreover, the cross-validation BIC actually shows an increase (worsening performance) after five clusters for both secondand third-order polynomial trajectories (Fig. 3b).

The cross-validation $F_{0.95}$ offers a somewhat different perspective to the traditional $F_{0.95}$ in that it suggests that first-order polynomial trajectories produce weaker cluster assignments than higher orders (Fig. 3d). However, like its traditional counterpart, the cross-validation $F_{0.95}$ does not effectively discriminate among higher polynomial orders, or among numbers of clusters.

In summary, traditional and cross-validation BIC and $F_{0.95}$ diagnostics provide different perspectives on model selection. Third- and second-order polynomial trajectories are supported by traditional and crossvalidation BIC, respectively, so the latter appears to favor less complex models. Also, while evidence for the choice of number of clusters is not conclusive, the crossvalidation BIC provides additional backing for not exceeding five clusters with either second- or third-order polynomials. Finally, the cross-validation $F_{0.95}$, in conjunction with the traditional BIC, helps eliminate model specifications with linear trajectories from further consideration; $F_{0.95}$ values provide little further discrimination. Combining all available information on this obviously challenging data, we conclude that there is no support for using trajectories higher than third order or more than five clusters, and that while this model specification may be somewhat redundant, it is certainly appropriate for capturing the structure of the IFS forecasts of Hurricane Ike initialized at 0000 UTC 7 September 2008.

\section{3) INTERPRETATION OF CLUSTERING RESULTS}

The third-order, five-cluster regression mixture model estimated on these forecasts of Ike initialized at 0000 UTC 7 September 2008 produces a clearly interpretable cluster solution (Fig. 4). The mean trajectory of the westernmost cluster (red, 11 members) traverses the northwest Caribbean Sea just north of the Yucatan Peninsula. The mean trajectories of the two most populous clusters (magenta and dark blue; 13 members each) cross Cuba, stretching west-northwestward into the central Gulf of Mexico. These two trajectories 


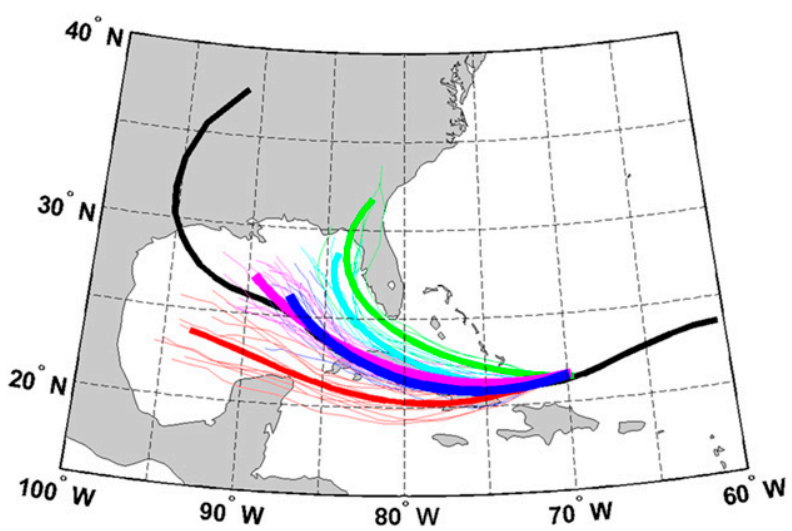

FIG. 4. ECMWF IFS ensemble forecasts (thin lines) and clustermean trajectories (thick lines) from path clustering of Hurricane Ike IFS forecasts initialized at 0000 UTC 7 Sep. The cluster solutions use a third-order polynomial, five-cluster regression mixture model. The mean trajectories for the most populous clusters are thicker.

follow the observed track closely, with members of the magenta cluster generally tracking faster than members of the dark blue cluster. The mean trajectories of the two eastern clusters (cyan, 8 members; green, 5 members) show Ike first moving farther north and then turning northeastward toward Florida late in the forecast period.

The five cluster mean trajectories just described provide the forecaster with five representative tracks that Hurricane Ike might have taken between 0000 UTC 7 September and 0000 UTC 12 September, based on forecasts from the ECMWF IFS ensemble. The two central clusters have the largest populations (combined 26 out of 50 total) and similar mean trajectories. The westernmost and especially the two eastern clusters have fewer members, suggesting less likely tracks. This would indicate to a forecaster that a track similar to the magenta or dark blue mean trajectory is the most likely outcome. It would also indicate to the forecaster that each of the five mean trajectories has a nonnegligible likelihood (each cluster has more than five members, i.e., $10 \%$ of the total; Table 6 ).

Using the model parameter estimates, the control forecast and the high-resolution deterministic forecast can each be assigned to a cluster a posteriori. In this example case, both are assigned to the magenta cluster, increasing the confidence that the central clusters capture the most probable track outcomes. Indeed, the 120-h observed track between 0000 UTC 7 September and 0000 UTC 12 September is also assigned to the magenta cluster a posteriori (Table 6).

We note that the similarity between the mean trajectories of the two most populous clusters is further evidence that a more parsimonious model specification (e.g., with four clusters) may have been equally effective for clustering this set of IFS forecasts (see above). However, we next show that the specification with thirdorder polynomial trajectories and five clusters has another critical advantage: it is complex enough to accommodate the clustering of IFS forecasts for all initialization times of all three storms considered in our study.

\section{b. Clustering 30 IFS ensemble forecasts from three storms}

Having demonstrated that third-order polynomial trajectories and five clusters suffice, perhaps with some redundancy, to capture the variability of the example IFS forecasts of Ike just described, we now show that this model specification is reasonable for the set of 30 ensemble forecasts from Sinlaku, Noul, and Ike. To do this, we examine the traditional and cross-validation versions of BIC and $F_{0.95}$ on all 30 initialization times, balancing goodness of fit and parsimony in evaluating which specifications perform satisfactorily across the board.

Both the traditional and cross-validation $F_{0.95}$ (shown in the online supplemental material) indicate that using first-order trajectories typically produces a larger fraction of weaker cluster assignments. This is especially true for forecasts of Sinlaku and Noul. However, similar to what was observed in section $6 \mathrm{a}$, neither traditional nor crossvalidation $F_{0.95}$ values provide substantial discrimination beyond eliminating first-order specifications.

The traditional (Fig. 5) and cross-validation (Fig. 6) BIC are more useful than the traditional and crossvalidation $F_{0.95}$ in selecting model specifications for our data. Traditional BIC values show that, throughout the forecasts and the range considered for the number of clusters, there is a substantial advantage in using secondorder versus first-order trajectories. Furthermore, for some forecasts, there is an additional, sizeable advantage in increasing the polynomial to third order. This is especially true for the later initialization times of Noul. Importantly, the traditional BIC does not exhibit a decrease beyond third-order polynomials for any of the 30 IFS forecasts, which suggests that this order is sufficient for capturing the complexity of all 30 sets of 120 -h forecast tracks for the three storms considered (Fig. 5).

Similar to the observations in section $6 \mathrm{a}$, the traditional BIC appears less helpful in discriminating among different numbers of clusters across all 30 datasets. Focusing on the curves corresponding to third-order polynomials (in red), we see that the traditional BIC decreases monotonically with the number of clusters in all panels of Fig. 5. This means that the cluster solutions keep improving up to at least seven clusters. However, for the majority of the 30 IFS forecasts, the rate of decrease in BIC tapers off after 5 clusters. The 


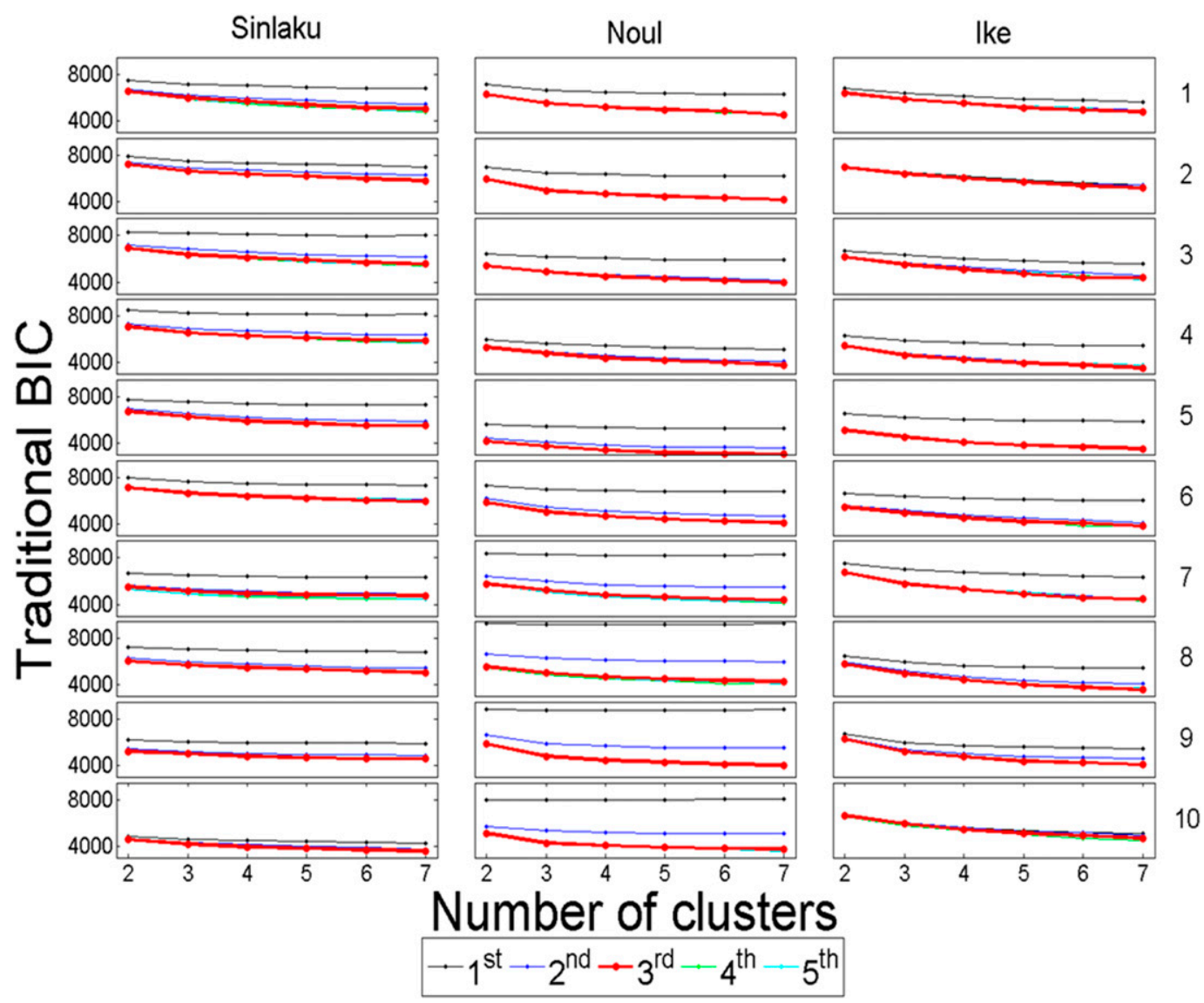

FIG. 5. Traditional BIC for various polynomial orders (line color, see legend) and number of clusters (horizontal axis) for all IFS forecasts of Sinlaku, Noul, and Ike. The 10 panels reported vertically for each storm represent chronologically ordered initialization times.

cross-validation BIC (Fig. 6) favors somewhat more parsimonious model specifications than the traditional BIC, similar to the outcome for the example in Section 6a. For some forecasts, the cross-validation BIC points toward use of first- or second-order polynomial trajectories (e.g., early forecasts for Ike), while for other forecasts (e.g., late forecasts for Noul) it supports the need for higher polynomial orders, and the variability in crossvalidation values (not reported in Fig. 6, but shown in Fig. 3b) imposes caution in drawing conclusions. An important result is that the cross-validation BIC increases (worse model fit) for polynomial orders beyond third for the majority of forecasts. These crossvalidation BICs reinforce the evidence provided by the traditional BIC, which did not show a decrease beyond third-order polynomials for any of the 30 IFS forecasts. Both the traditional and the cross-validation diagnostics, the latter trending more toward parsimony than the former, indicate that there is no need for polynomial trajectories of higher than third order in any of the forecasts considered.
The more parsimonious nature of the cross-validation BIC appears also when evaluating the optimal number of clusters. Focusing again on the curves corresponding to third-order polynomials (in red), we note that, while the traditional BIC decreases monotonically between 2 and 7 clusters for all 30 IFS forecasts-tapering off after 5 clusters in most (Fig. 5) - the cross-validation BIC usually increases monotonically with cluster number or reaches a minimum value between 3 and 5 clusters (Fig. 6). This signal represents an important complement to the information provided by the traditional diagnostic.

The ECMWF IFS forecasts considered here incorporate a wide diversity in storm intensity and life cycle stage, as well as in the shape of the forecast paths. Nevertheless, the overarching conclusion we can draw based on the evidence offered by BIC and $F_{0.95}$ in their traditional and cross-validation versions, is that a regression mixture model using third-order polynomial trajectories and five clusters amply captures the variation and complexity of all forecasts. Third-order 


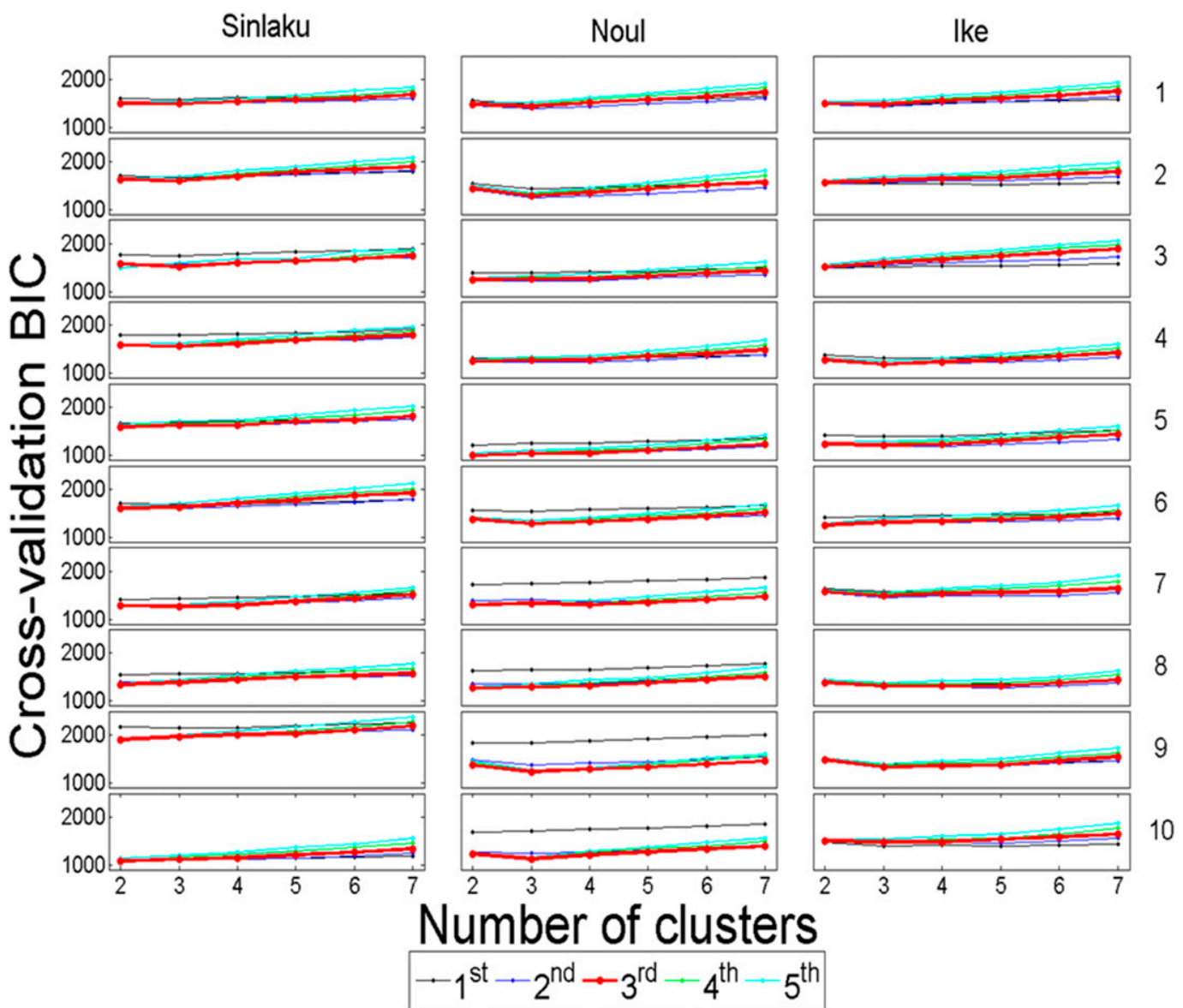

FIG. 6. Cross-validation BIC for various polynomial orders (line color, see legend) and number of clusters (horizontal axis) for all IFS forecasts of Sinlaku, Noul, and Ike. The 10 panels reported vertically for each storm represent chronologically ordered initialization times.

polynomials are backed by both traditional and crossvalidation diagnostics, and the choice of five clusters emerges by combining evidence from the traditional $\mathrm{BIC}$ and the more parsimonious cross-validation BIC.

\section{Interpretation of clustering results from IFS track forecasts}

The three TCs examined here have very different tracks and structural evolutions (tropical strengthening, tropical landfall/weakening, and extratropical transition). We now examine the usefulness of our clustering methodology in characterizing these evolutions. To this end, we explore the clustering results focusing on a single initialization time from each TC.

\section{a. Typhoon Sinlaku (2008)}

For Typhoon Sinlaku, the third-order, five-cluster specification produces interpretable partitions of IFS ensemble members based on both speed and direction of motion (Fig. 7, Table 5). Note that the colors of clustermean trajectories in Figs. 7-9 map to the cluster numbers in Tables 5-7 as follows: $1=$ red, $2=$ magenta, $3=$ dark blue, $4=$ cyan, and $5=$ green; number progression is from westernmost to easternmost cluster-mean trajectory, so consecutive cluster numbers are adjacent trajectories.

As an example, consider the IFS forecasts initialized at 0000 UTC 16 September, (Fig. 7d); at this time, Sinlaku was located northeast of Taiwan and was drifting to the east as a $45-\mathrm{kt}\left(23 \mathrm{~m} \mathrm{~s}^{-1}\right)$ tropical storm (Fig. 2a, location 5 in the Philippine Sea). In the red mean trajectory (westernmost cluster), Sinlaku moves farthest to the north, staying west of Kyushu and moving into the Sea of Japan before making landfall in northwest Honshu. In the magenta, dark blue, and cyan mean trajectories, Sinlaku takes a similar track toward Kyushu early in the forecast. However, later in the forecast the three clusters diverge: the magenta mean trajectory moves slowest and continues to the northeast, while the cyan 

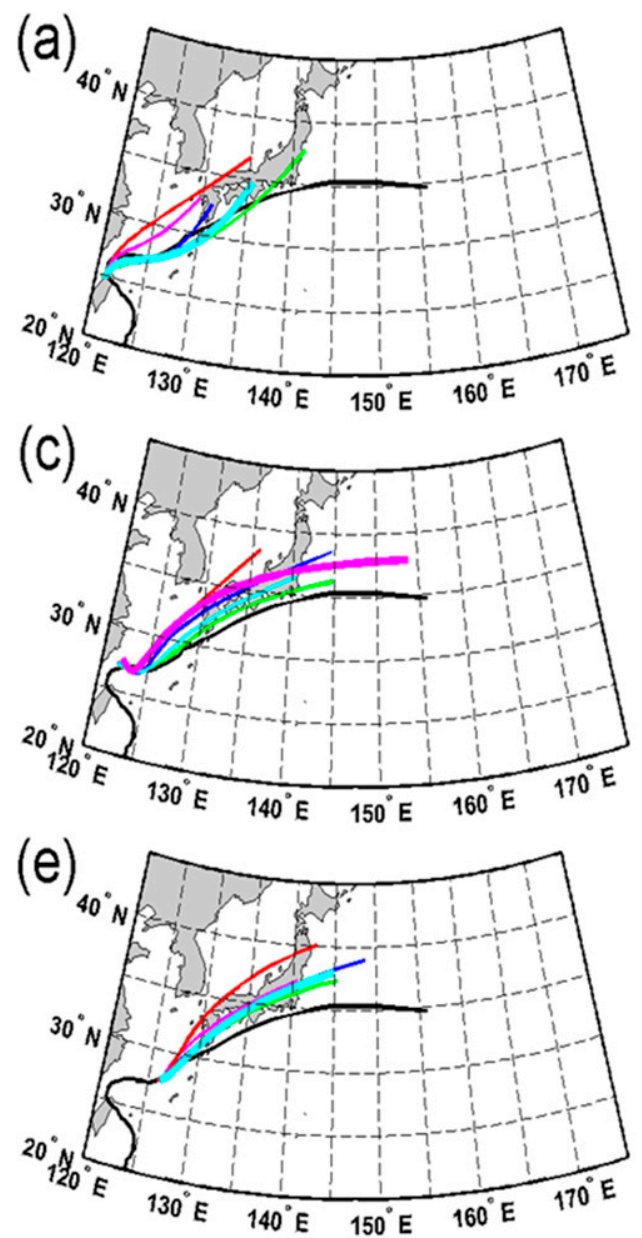
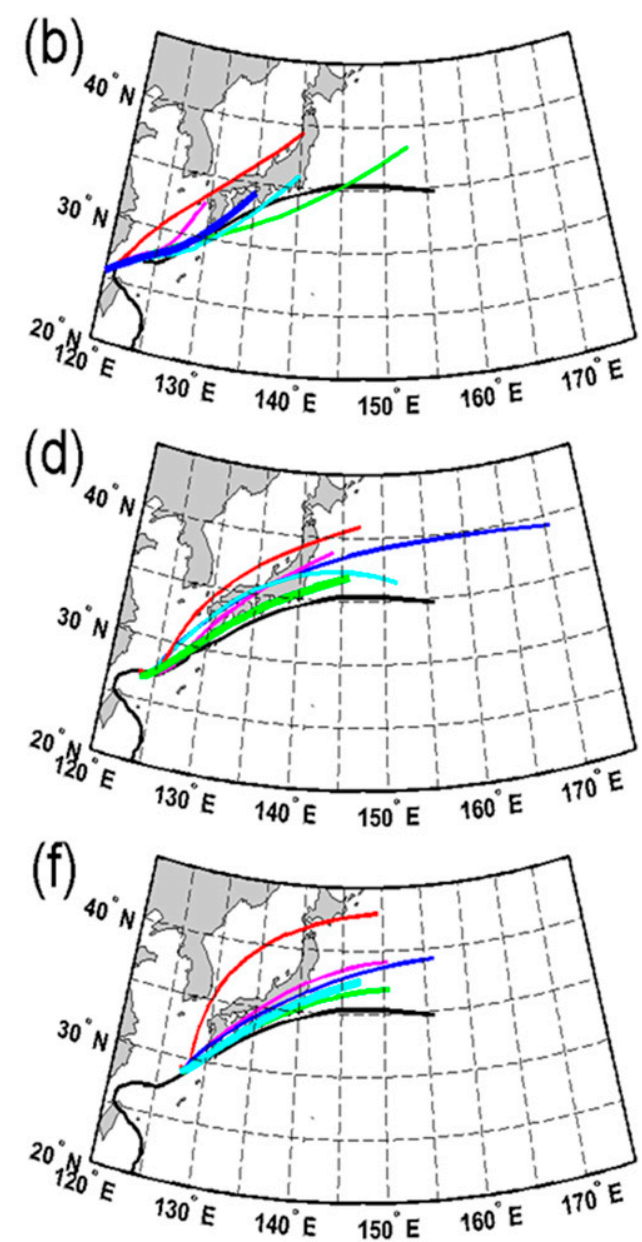

FIG. 7. Cluster mean trajectories for Typhoon Sinlaku from (a) 0000 UTC 14 Sep, (b) 1200 UTC 14 Sep, (c) 1200 UTC $15 \mathrm{Sep}$, (d) 0000 UTC $16 \mathrm{Sep}$, (e) 0000 UTC $17 \mathrm{Sep}$, and (f) 1200 UTC $17 \mathrm{Sep}$. The mean trajectory of the most populous cluster is represented by a thicker line. The observed track is shown in black. The colors of cluster-mean trajectories map to the cluster numbers in Tables 5-7 as follows: $1=$ red, $2=$ magenta, $3=$ dark blue, $4=$ cyan, and $5=$ green. The progression is from westernmost to easternmost mean trajectory, so consecutive cluster numbers are adjacent trajectories.

one moves somewhat more rapidly toward the northnortheast across Japan, turning eastward after exiting land. The dark blue mean trajectory falls between these two, but its members move Sinlaku far more rapidly than those in all other clusters, crossing Japan and racing eastward over the North Pacific. These four mean trajectories are all well north of the observed track (in black). The green mean trajectory (easternmost cluster) is closer to the observed track, albeit still somewhat too far to the north. Notably, the green cluster is (marginally) the most populous (13 of 50 members; Table 5).

Similar comments apply to other forecast times (Fig. 7). Although the majority of the mean trajectories are consistently to the left of the observed track, especially for the later forecasts (Figs. 7c-f), the third-order, five-cluster specification satisfactorily captures the spread in speed and direction of motion among the 50 IFS ensemble members for each initialization time.

\section{b. Hurricane Ike (2008)}

In the case of Hurricane Ike's forecasts, the thirdorder, five-cluster specification once again produces mean trajectories that are distinct and capture the spread of the IFS forecast tracks. This is true for all initialization times, but the clustering is demonstrated to be especially effective in the case of IFS forecasts initialized at 1200 UTC 5 September (Fig. 8b; location 4 in Fig. 2b). At this time, in the red mean trajectory Ike moves the farthest south, then tracks due west, making landfall on the Yucatan Peninsula late in the forecast period. The other mean trajectories are progressively 
TABLE 5. Summary information on the clustering of IFS forecasts of Typhoon Sinlaku, using third-order polynomial trajectories and five clusters. (last five columns) Distribution of the IFS forecasts across clusters. (second, fourth, and fifth columns) Clusters to which the observed track and the deterministic and control forecast track are assigned. (third column) Most populous cluster. Bolded initialization times are those for which mean trajectories are shown in Fig. 7. The colors of cluster-mean trajectories in Fig. 4 and Figs. 7-9 map to the cluster numbers here as follows: $1=$ red, $2=$ magenta, $3=$ dark blue, $4=$ cyan, and $5=$ green. The progression is from westernmost to easternmost mean trajectory, so consecutive cluster numbers are adjacent trajectories.

\begin{tabular}{cccccrrrrr}
\hline \hline Initial time & Observed cluster & Most populous cluster & Deterministic cluster & Control cluster & 1 & 2 & 3 & 4 & 5 \\
\hline 0000 UTC 14 Sep & 3 & 4 & 3 & 4 & 8 & 13 & 10 & 14 & 5 \\
1200 UTC 14 Sep & 3 & 3 & 3 & 3 & 12 & 5 & 20 & 12 & 1 \\
0000 UTC 15 Sep & 5 & 2 & 5 & 4 & 5 & 16 & 11 & 13 & 5 \\
1200 UTC 15 Sep & 5 & 2 & 5 & 3 & 9 & 11 & 10 & 10 & 10 \\
0000 UTC 16 Sep & 4 & 5 & 4 & 2 & 10 & 12 & 3 & 12 & 13 \\
1200 UTC 16 Sep & 5 & 1 & 3 & 3 & 15 & 12 & 10 & 6 & 7 \\
0000 UTC 17 Sep & 3 & 4 & 5 & 10 & 8 & 12 & 13 & 7 \\
1200 UTC 17 Sep & 5 & 4 & 5 & 5 & 3 & 9 & 6 & 20 & 12 \\
0000 UTC 18 Sep & 5 & 3,4 & 1 & 4 & 9 & 10 & 11 & 11 & 8 \\
0000 UTC 19 Sep & 2 & 5 & 4 & 9 & 10 & 7 & 10 & 14 \\
\hline
\end{tabular}

farther to the right of this track, with Ike moving through the Yucatan Channel (magenta), over western Cuba (dark blue), into the straits of Florida (cyan), and making landfall in south Florida (green). The dark blue cluster, with mean trajectory crossing western Cuba, is the one to which the observed track is assigned; in this case, dark blue is also the most populous cluster (16 out of 50 members; Table 6). In contrast, the red and green clusters are the least populous (six members each). The third-order, five-cluster specification effectively captures the variation in IFS ensemble members, providing information on the potential tracks of Ike (cluster mean trajectories) and the likelihood of each such track (population of each cluster).

The five mean trajectories from 1200 UTC 5 September (Fig. 8b) show substantial spread in the direction of motion but less difference in speed of motion, indicating that the primary uncertainty in the track of Ike at this time was in direction. However, at other initialization times the five mean trajectories also show substantial spread in speed of motion. For example, while the magenta and dark blue trajectories from the 0000 UTC 7 September initialization (Fig. 8d) are similar, the ensemble tracks are primarily partitioned between these two clusters based on speed of motion: at $120 \mathrm{~h}$, the magenta cluster is substantially closer to landfall than the dark blue cluster (Fig. 8d; see also Fig. 4).

\section{c. Typhoon Noul (2015)}

In the case of Typhoon Noul's forecasts, the thirdorder, five-cluster specification produces mean trajectories in which the storm makes landfall at different locations in the northern Philippines, and mean trajectories in which it remains to the east of the Philippines (e.g., Fig. 9c). In the later forecasts, the clustering solutions separate scenarios in which Noul recurves sharply toward the northeast, and thus out to sea, and scenarios in which it recurves more gradually and tracks across Japan (e.g., Fig. 9f). The IFS forecasts initialized at 1200 UTC 5 May (Fig. 9b) again illustrate how clustering partitions can capture distinctions in both direction and speed of motion. At this time Noul was a minimal typhoon located near Yap, Micronesia (location 4 in Fig. 2a). The mean trajectories of the (marginally) most populous dark blue cluster (14 members; Table 7) and the cyan cluster (9 members) both track toward the northern tip of Luzon while beginning to recurve. However, the cyan mean moves faster and undergoes more recurvature than the dark blue mean through $120 \mathrm{~h}$ (Fig. 9b). The mean trajectory of the green cluster (smallest; 2 members) recurves well east of the Philippines, thus, indicating a possible but unlikely forecast scenario. The mean trajectories of the westernmost red (13 members) and magenta (12 members) clusters take similar tracks toward central Luzon; however, the IFS members in the magenta cluster move substantially faster. Clustering solutions able to render direction and speed of motion patterns are observed at all other forecast times.

\section{d. Forecast clusters and observed and deterministic tracks}

For each initialization time and for each storm, the clustering solution is found by applying a third-order, fivecluster regression mixture model on the data from the corresponding 50 IFS forecasts (section 5). For each initialization time and for each storm, we also extract a 120-h segment of the observed track, as well as the deterministic and the control forecasts. Based on the model estimates, the observed track, deterministic forecast, and control forecast can each be assigned to a cluster a posteriori, 

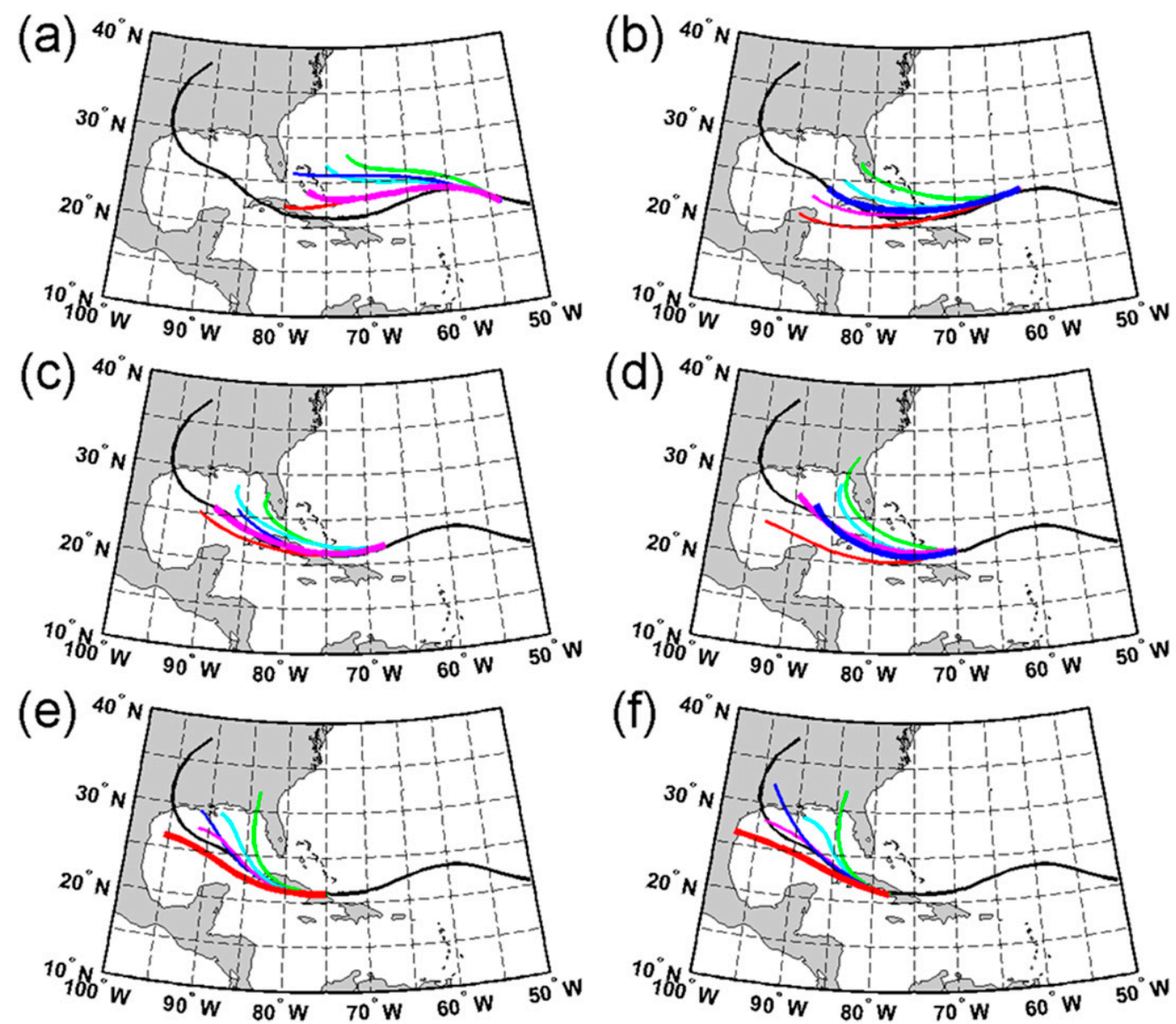

FIG. 8. Cluster mean trajectories for Hurricane Ike from (a) 0000 UTC 4 Sep, (b) 1200 UTC 5 Sep, (c) 1200 UTC 6 Sep, (d) 0000 UTC 7 Sep, (e) 0000 UTC 8 Sep, and (f) 1200 UTC 8 Sep. The mean trajectories of the most populous cluster(s) are represented by thicker line(s). The observed track is shown in black.

providing another means to evaluate the solution. We report the distribution of the 50 IFS forecasts across clusters, the most populous cluster, and the clusters to which the observed track, the deterministic forecast, and the control forecast are assigned, for Typhoon Sinlaku (Table 5), Hurricane Ike (Table 6), and Typhoon Noul (Table 7) across initialization times. In the majority of cases, the observed track is either in the most populous cluster or in an adjacent one (moving west to east).

These results confirm that the third-order, five-cluster model specification provides interpretable partitions of the 50 ECMWF IFS forecasts at all initialization times for each of the three tropical cyclones based on both speed and direction of motion.

\section{e. Track error characteristics of dominant and observed forecast clusters}

To gauge the effectiveness of clustering as a means to provide forecast guidance for these three storms, we compare the along-track (speed) and across-track (direction) errors of the IFS overall ensemble mean track (Fig. 10, blue) with the same errors calculated for the mean trajectory of the dominant (most populous) cluster at 48-72-h forecast lead time (Fig. 10a, red), and for the mean trajectory of the observed cluster at both 48-72and 96-120-h lead times (Figs. 10b,c, red). Errors are calculated relative to the postanalysis best track issued by the National Hurricane Center (NHC) for Hurricane Ike, or by the Joint Typhoon Warning Center (JTWC) for Typhoon Sinlaku. For Typhoon Noul, the postanalysis best track was not yet available, so the operational track was used. To improve the reliability of our comparisons we restrict attention to initialization times in which $80 \%$ or more of the ensemble members still have the storm present at the forecast lead time used in the error calculation for each storm. Moreover, we consider the dominant cluster only when it has at least 4 more members than the next most populous cluster.

For the three storms examined, the dominant cluster mean trajectory and overall ensemble mean track 


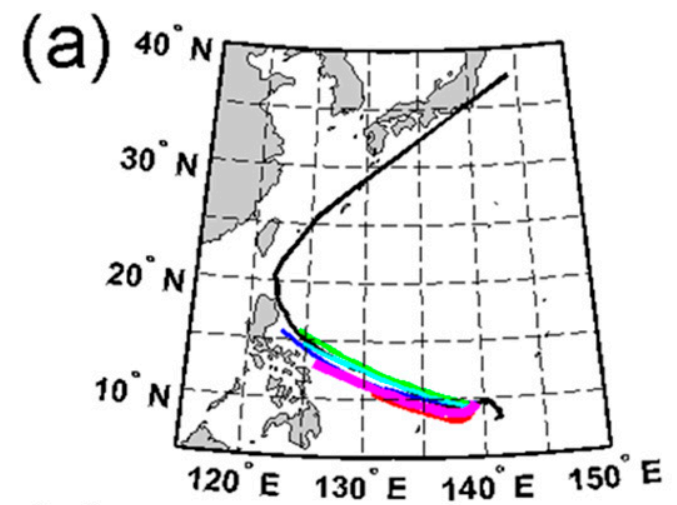

(c)

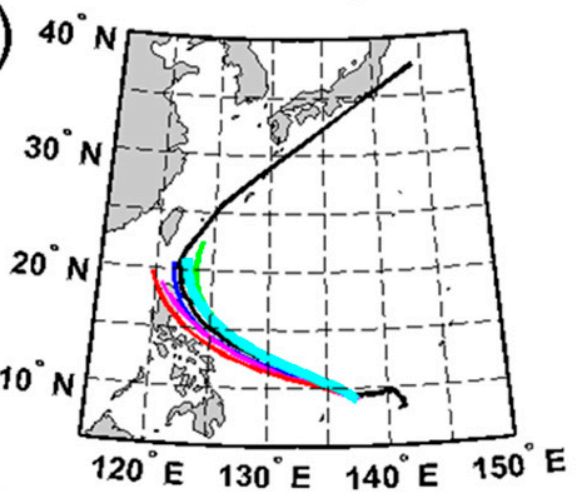

(e)

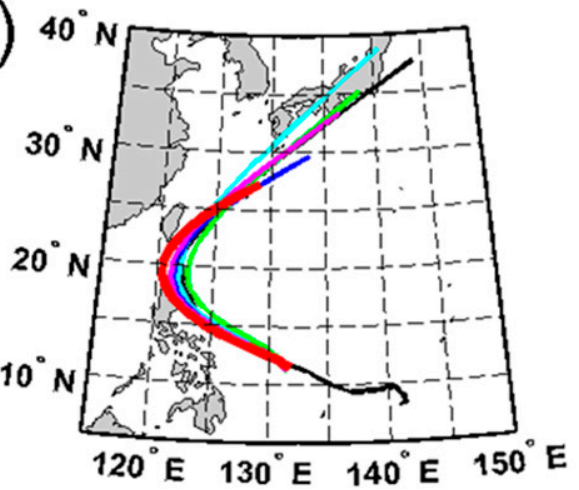

(b)

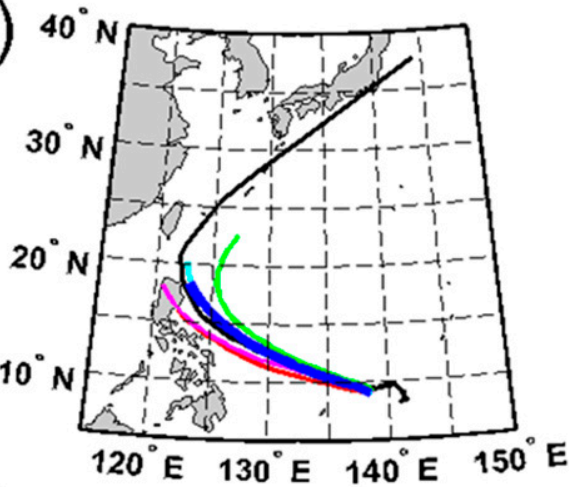

(d)

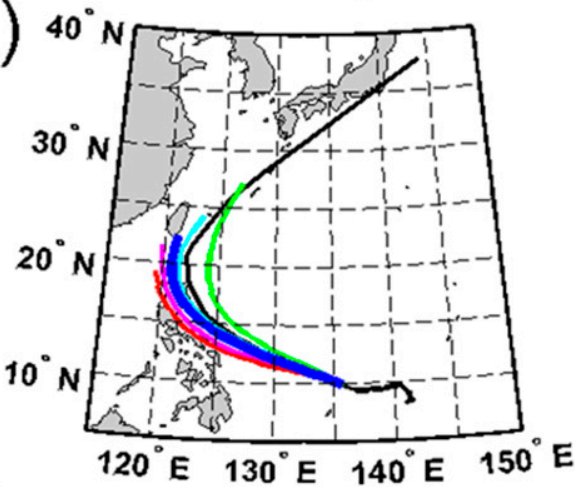

(f)

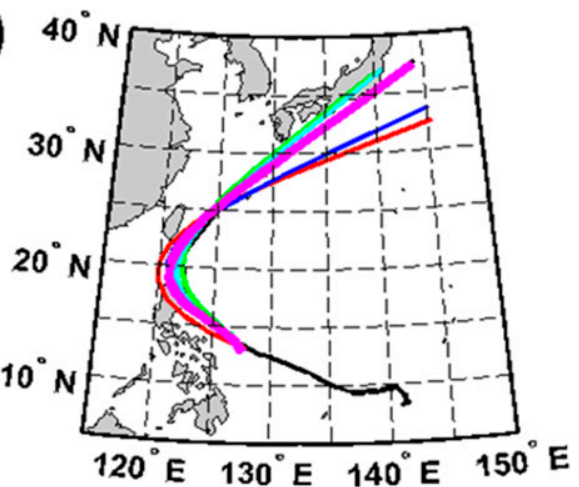

FIG. 9. Cluster mean trajectories for Typhoon Noul from (a) 1200 UTC 4 May, (b) 1200 UTC 5 May, (c) 0000 UTC 6 May, (d) 1200 UTC 6 May, (e) 1200 UTC 7 May, and (f) 1200 UTC 8 May. The mean trajectory of the most populous cluster is represented by a thicker line. The observed track is shown in black.

appear to have similar performance along both error dimensions (Fig. 10a). However, the observed cluster mean trajectory tends to have smaller along-track and across-track errors for both 48-72- and 96-120-h lead times (Figs. 10b,c). Although the shift in error distributions is not striking, it shows promise for the proposed methodology. Furthermore, substantial differences in synoptic evolution are observed among clusters, suggesting that track clustering captures variations in forecasts of the large-scale flow. We are in the process of greatly expanding our forecast storm database and examining the synoptic partitions resulting from the use of mixture-based path clustering on a larger collection of storm events. A more extensive exploration of the potential of the proposed methodology is thus forthcoming.

\section{Conclusions}

We examine 120-h ECMWF IFS track forecasts obtained at 10 initialization times for each of three TCs: Typhoons Sinlaku (2008), Typhoon Noul (2015), and Hurricane Ike (2008). On each of the 30 datasets, we implement path clustering with a regression mixture model specified in terms of order of polynomial 
TABLE 6. Summary information on the clustering of IFS forecasts of Hurricane Ike, using third-order polynomial trajectories and five clusters. Bolded initialization times are those for which mean trajectories are shown in Fig. 8. The colors of cluster-mean trajectories in Fig. 8 map to the cluster numbers as in Tables 5 and $7(1=$ red, $2=$ magenta, $3=$ dark blue, $4=$ cyan, and $5=$ green).

\begin{tabular}{ccccrrrrrr}
\hline \hline Initial time & Observed cluster & Most populous cluster & Deterministic cluster & Control cluster & 1 & 2 & 3 & 4 \\
\hline 0000 UTC 4 Sep & 1 & 2 & 2 & 2 & 12 & 13 & 9 & 12 & 4 \\
1200 UTC 4 Sep & 2 & 2 & 2 & 2 & 10 & 18 & 9 & 6 & 7 \\
0000 UTC 5 Sep & 2 & 4 & 2 & 2 & 11 & 12 & 9 & 13 & 5 \\
1200 UTC 5 Sep & 3 & 3 & 3 & 3 & 6 & 12 & 16 & 10 & 6 \\
0000 UTC 6 Sep & 4 & 2,4 & 4 & 4 & 11 & 13 & 2 & 13 & 11 \\
1200 UTC 6 Sep & 2 & 2 & 2 & 3 & 9 & 12 & 11 & 11 & 7 \\
0000 UTC 7 Sep & 3 & 2,3 & 3 & 3 & 11 & 13 & 13 \\
1200 UTC 7 Sep & 4 & 4 & 2 & 5 & 7 & 12 & 10 & 14 & 7 \\
0000 UTC 8 Sep & 1 & 1 & 2 & 2 & 12 & 11 & 10 & 10 & 7 \\
1200 UTC 8 Sep & 2 & 1 & 2 & 16 & 13 & 5 & 10 & 6 \\
\hline
\end{tabular}

trajectories and number of clusters. To select an appropriate model, we use two diagnostics: the Bayesian information criterion (BIC) and the strength of probabilistic cluster assignments measured by $F_{0.95}$. In addition, we formulate and compute cross-validation versions of these traditional diagnostics.

We find that, for the data analyzed in this study, the BIC provides a more useful diagnostic for discriminating among model specifications than $F_{0.95}$, and that the cross-validation BIC tends to support more parsimonious specifications (lower-order polynomials, fewer clusters) than the traditional BIC, thus, demonstrating the importance of considering both versions of the diagnostic in model selection. We also find that, across the 30 IFS forecast periods considered, it is easier to select the order of the polynomial trajectories than the number of clusters. This opaqueness to number of clusters confirms what is apparent from visual inspection of the forecasts (e.g., Fig. 1); namely, that the ensemble member tracks often spread without showing obvious "lumping" into subgroups. As our analysis shows, this does not negate the utility of path clustering as a way to estimate a small number of distinct and interpretable mean trajectories-indeed, it makes this analysis more valuable-but it does render the choice of how many such trajectories to use somewhat arbitrary.

We evaluate mixture model specifications with polynomial order ranging from first to fifth, and number of clusters ranging from two to seven. Because IFS forecasts for various initialization times and storms differ in their level of complexity, as do the observed storm evolutions, optimal model specifications vary among initialization times. However, in most cases, a model with third-order polynomial trajectories and five clusters resulted in best or near-best performance according to our diagnostics. Most importantly, while this specification may have been slightly redundant in some cases, no case supported the choice of a more complex model. Thus, we can safely select third-order, five-cluster solutions to capture the variability of all 30 IFS forecasts in this study. In fact, these solutions represent interpretable partitions of direction and speed of motion of all of the forecast tracks, synthesizing a large set of ensemble members into a small number of representative and distinct mean trajectories. Moreover, the clusters to which observed tracks are posteriorly assigned are

TABLE 7. Summary information on the clustering of IFS forecasts of Typhoon Noul, using third-order polynomial trajectories and five clusters. Bolded initialization times are those for which mean trajectories are shown in Fig. 9. The colors of cluster-mean trajectories in Fig. 9 map to the cluster numbers as in Tables 5 and $6(1=$ red, $2=$ magenta, $3=$ dark blue, $4=$ cyan, and $5=$ green $)$.

\begin{tabular}{cccccrrrrr}
\hline \hline Initial time & Observed cluster & Most populous cluster & Deterministic cluster & Control cluster & 1 & 2 & 3 & 5 \\
\hline 0000 UTC 4 May & 4 & 3 & 3 & 3 & 2 & 11 & 13 & 12 & 12 \\
1200 UTC 4 May & 5 & 2 & 4 & 4 & 8 & 17 & 2 & 14 & 9 \\
0000 UTC 5 May & 4 & 2,5 & 4 & 5 & 10 & 12 & 7 & 9 & 12 \\
1200 UTC 5 May & 4 & 3 & 2 & 2 & 13 & 12 & 14 & 9 & 2 \\
0000 UTC 6 May & 3 & 4 & 3 & 3 & 6 & 10 & 12 & 13 & 9 \\
1200 UTC 6 May & 3 & 3 & 3 & 3 & 8 & 16 & 12 & 7 \\
0000 UTC 7 May & 3 & 2 & 5 & 3 & 11 & 17 & 11 & 8 & 3 \\
1200 UTC 7 May & 2 & 1 & 4 & 2 & 13 & 11 & 10 & 5 & 11 \\
0000 UTC 8 May & 4 & 3 & 4 & 4 & 9 & 18 & 9 & 10 \\
1200 UTC 8 May & 4 & 2 & 2 & 9 & 17 & 8 & 9 & 7 \\
\hline
\end{tabular}


a.

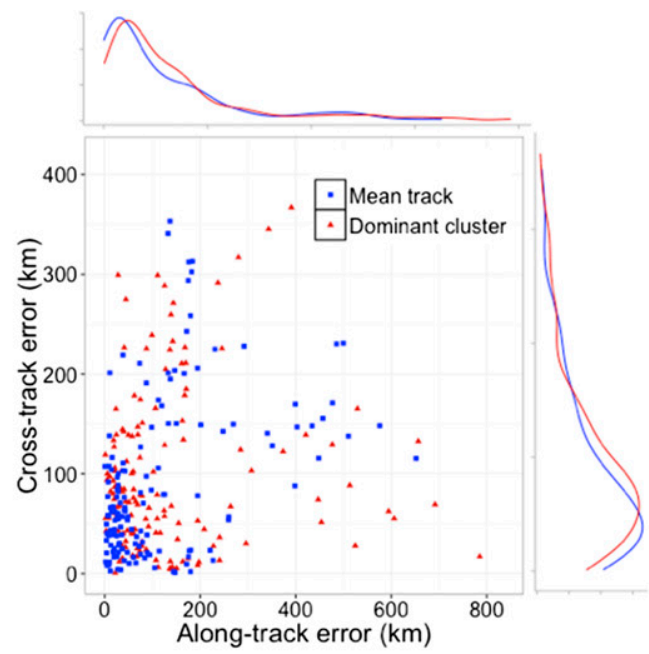

b.

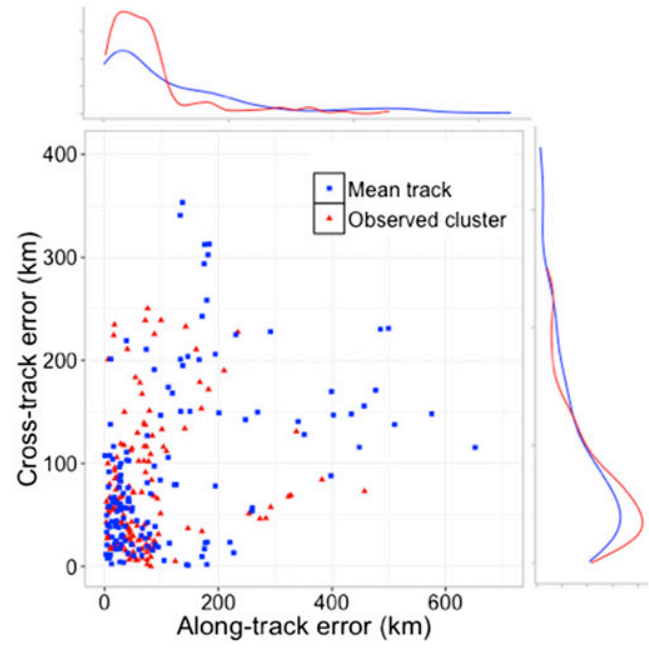

c.

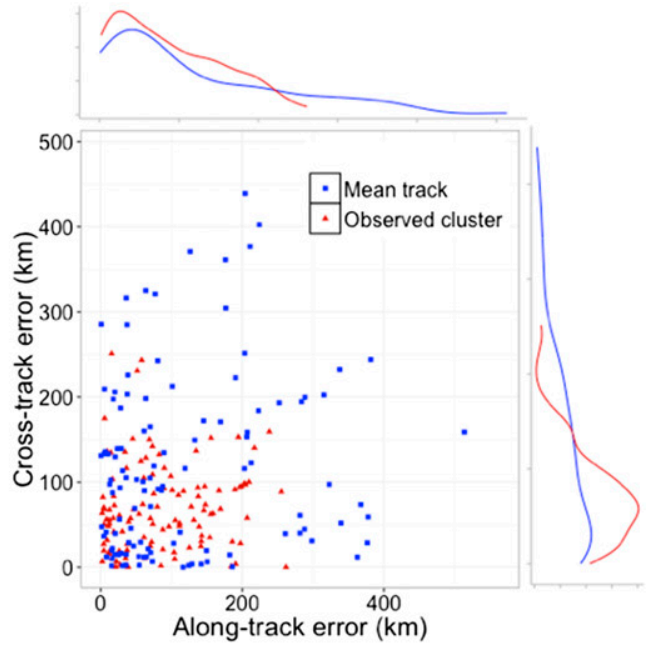

FIG. 10. Evaluation of along-track (speed) and across-track (direction) error of the overall ensemble mean track (blue) and (a) the dominant cluster mean trajectory for 48-72-h forecast lead times, (b) the observed cluster mean trajectory for 48-72-h lead times, and (c) the observed cluster mean trajectory for 96-120-h lead times (all in red). Density plots are provided on each axis to help in visually comparing distributions. frequently the most populous clusters, or are adjacent to the most populous cluster. We are assessing the robustness of this result as we increase the number of storm events analyzed.

We are also in the process of examining the thirdorder, five-cluster model specification for a larger set of storms to evaluate its utility more broadly, as we believe that the path clustering approach used here could have significant operational impacts on TC forecasting. Notably, even if the model specifications were reevaluated for every IFS forecast, the computational burden of this approach would be quite acceptable (see the appendix for some details).

Our approach could also be extended to other timedependent forecast metrics whose temporal evolution can be summarized as a low-dimensional path. For example, we are examining the utility of clustering global ensemble forecasts represented as paths in the cyclone phase space (CPS), which captures the storm structure evolution (Hart 2003; Evans and Hart 2003). Furthermore, our approach can be compared to, and used in conjunction with, other types of clusteringincluding environmental synoptic clustering (e.g., Harr et al. 2008; Keller et al. 2011, 2014). The use of complementary methods of ensemble forecast clustering has the potential to improve forecasting of both stormscale and environmental-scale phenomena. In cases of extratropical transition, the storm structure and intensity, as well as its environment, are critical to the storm's ultimate evolution (and potential reintensification as an extratropical system; e.g., Hart et al. 2006; Keller et al. 2014) and potentially also to the genesis and evolution of midlatitude storm systems. Thus, integrating information on storm track and structure with environmental information may provide new insights into the sources of uncertainty associated with ET forecasts. These sources of uncertainty, in turn, affect future storm evolution (complete ET or decay, reintensification potential) and the development of midlatitude cyclones downstream of the ET event itself.

Finally, we point out that functional data analysis techniques provide an appealing and effective alternative approach for clustering forecast tracks, finding robust summaries and evaluating their uncertainty (e.g., M. Hubert, P. J. Rousseeuw, and P. Segaert, 2015, personal communication; Ieva and Paganoni, 2013; LópezPintado and Jornsten, 2007; López-Pintado et al. 2014; Whitaker et al. 2013; Mirzargar et al. 2014; Ferstl et al. 2016). As an extension to our work, we are currently exploring the possibility of using data depth in multivariate functional data to estimate the uncertainty associated with mean forecast tracks produced by path clustering with regression mixture models. 
Acknowledgments. We are grateful to Mr. Casey Webster, Dr. Julia Keller, and Dr. Sarah Jones for many helpful conversations. We thank Dr. Chuck Pavloski and Mr. Chad Bahrmann for their excellent programming assistance and database management. The comments of three anonymous reviewers led to substantial improvements in the manuscript. The ECMWF ensemble forecasts were obtained via the TIGGE database. This research was supported by the National Science Foundation under Grant ATM-1322532.

\section{APPENDIX}

\section{Technical Details on Path Clustering with Polynomial Regression Mixtures}

Mixture-based clustering views observations as draws from a mixture of probability distributions (components), each representing a cluster. In most applications the mixture is assumed to comprise a finite number of components, and the components are assumed to be Gaussian about univariate, multivariate, or more complex means (in our case, polynomial trajectories in the 2D space of longitude and latitude). Identification of the component from which each observation is most likely to have been drawn produces cluster assignments.

We have $n$ tracks $\mathbf{Y}=\left\{\mathbf{y}_{1}, \mathbf{y}_{2}, \ldots, \mathbf{y}_{n}\right\}$, with each $\mathbf{y}_{i}$ comprising $n_{i}$ points in $\mathbb{R}^{2}$ corresponding to times $t_{i}$. Each $\mathbf{y}_{i}$ is, hence, a matrix of size $n_{i} \times 2$, which we represent as a $2 n_{i} \times 1$ vector by stacking the two columns (i.e., $\mathrm{y}_{i}=\left[y_{1 i}, y_{2 i}\right]^{\mathrm{T}}$ ). The $\mathbf{y}_{i} \mathrm{~s}$ are regarded as draws from a mixture of $K$ components. The relationship between $\mathbf{y}_{i}$ and $t_{i}$ is modeled as a component-specific $q$ th-order polynomial regression with additive Gaussian error:

$$
\mathbf{y}_{i}=\mathbf{T}_{i} \boldsymbol{\beta}_{k}+\boldsymbol{\epsilon}_{i} \quad \boldsymbol{\epsilon}_{i} \sim N\left(0, \boldsymbol{\Sigma}_{k}\right),
$$

where $\mathbf{T}_{i}$ is a matrix of size $2 n_{i} \times 2(q+1)$ such that

$$
\mathbf{T}_{i}=\left[\begin{array}{cc}
\mathbf{X}_{i} & 0 \\
0 & \mathbf{X}_{i}
\end{array}\right] \text { where } \quad \mathbf{X}_{i}=\left[\begin{array}{ccccc}
1 & t_{i 1} & t_{i 1}^{2} & \cdots & t_{i 1}^{q} \\
\vdots & \vdots & \vdots & \cdots & \vdots \\
1 & t_{i n i} & t_{i n i}^{2} & \cdots & t_{i n i}^{q}
\end{array}\right]
$$

Here, for the $k$ th component, $\boldsymbol{\beta}_{k}=\left[\beta_{1 k}, \beta_{2 k}\right]$ is the regression coefficient matrix of size $(q+1) \times 2$, which we represent as a $2(q+1) \times 1$ vector again stacking the two columns, and $\boldsymbol{\Sigma}_{k}$ is the error covariance matrix of size $2 n_{i} \times 2 n_{i}$. We constrain the latter to be of the following form:

$$
\boldsymbol{\Sigma}_{k}=\mathbf{D}_{k}=\left[\begin{array}{cc}
\sigma_{1 k}^{2} I & 0 \\
0 & \sigma_{2 k}^{2} I
\end{array}\right]
$$

The conditional density relating $\mathbf{y}_{i}$ to $t_{i}$ in the $k$ th component is thus

$$
p_{k}\left(\mathbf{y}_{i} \mid t_{i}, \boldsymbol{\theta}_{k}\right)=N\left(\mathbf{y}_{i} \mid \mathbf{T}_{i} \boldsymbol{\beta}_{k}, \mathbf{D}_{k}\right),
$$

where $\boldsymbol{\theta}_{k}=\left\{\beta_{1 k}, \beta_{2 k}, \sigma_{1 k}^{2}, \sigma_{2 k}^{2}\right\}$, and the overall mixture density relating $\mathbf{y}_{i}$ to $t_{i}$ is

$$
p\left(\mathbf{y}_{i} \mid t_{i}, \boldsymbol{\Theta}\right)=\sum_{k=1}^{K} \tau_{k} p_{k}\left(\mathbf{y}_{i} \mid t_{i}, \boldsymbol{\theta}_{k}\right)=\sum_{k=1}^{K} \tau_{k} N\left(\mathbf{y}_{i} \mid \mathbf{T}_{i} \boldsymbol{\beta}_{k}, \mathbf{D}_{k}\right)
$$

where $\boldsymbol{\Theta}=\left\{\tau_{1}, \ldots, \tau_{K}, \theta_{1}, \ldots, \theta_{K}\right\}$ and $\tau_{k}$ is the prior probability that $\mathbf{y}_{i}$ is generated by the $k$ th component $\left(\tau_{k} \geq 0, \Sigma \tau_{K}=1\right)$. It follows that the joint density of the forecast tracks in $\mathbf{Y}$ is

$$
p(\mathbf{y} \mid \mathbf{T}, \boldsymbol{\Theta})=\prod_{i=1}^{n} \sum_{k=1}^{K} \tau_{k} N\left(\mathbf{y}_{i} \mid \mathbf{T}_{i} \boldsymbol{\beta}_{k}, \mathbf{D}_{k}\right)
$$

and the log-likelihood function is

$$
\log p(\mathbf{y} \mid \mathbf{T}, \boldsymbol{\Theta})=\sum_{i=1}^{n} \log \sum_{k=1}^{K} \tau_{k} N\left(\mathbf{y}_{i} \mid \mathbf{T}_{i} \boldsymbol{\beta}_{k}, \mathbf{D}_{k}\right) .
$$

To use the EM algorithm to estimate parameters in $\boldsymbol{\Theta}$ and posterior probabilities (that each track was generated by each component), we need to expand the above framework to encompass "unobserved" data; namely, the component labels (cluster memberships) for each $\mathbf{y}_{i}$, say $z_{i} \in\{1$, $2, \ldots, K\}$. The joint density of $\mathbf{y}_{i}$ and $z_{i}$ can be written as

$$
p\left(\mathbf{y}_{i}, z_{i} \mid t_{i}, \boldsymbol{\Theta}\right)=\tau_{z_{i}} p_{z_{i}}\left(\mathbf{y}_{i} \mid t_{i}\right)=\tau_{z_{i}} N\left(\mathbf{y}_{i} \mid \mathbf{T}_{i} \boldsymbol{\beta}_{z_{i}}, \mathbf{D}_{z_{i}}\right) .
$$

Therefore the so-called complete data log-likelihood function can be written as

$$
L_{c}=\sum_{i=1}^{n} \log \tau_{z_{i}} N\left(\mathbf{y}_{i} \mid \mathbf{T}_{i} \boldsymbol{\beta}_{z_{i}}, \mathbf{D}_{z_{i}}\right) .
$$

The unobserved cluster memberships are regarded as hidden, and their densities are computed in the E step of the EM algorithm. In symbols, the posterior probabilities that each track was generated by each component are defined as $\omega_{i, k}=\operatorname{Pr}\left\{z_{i}=k \mid \mathbf{y}_{i}, t_{i}\right\}$. Their estimates are iteratively updated in the E step according to

$$
\hat{\omega}_{i, k}=\hat{\tau}_{k} N\left(\mathbf{y}_{i} \mid \mathbf{T}_{i} \hat{\boldsymbol{\beta}}_{k}, \hat{\mathbf{D}}_{k}\right),
$$

where $\hat{\tau}_{k}, \hat{\boldsymbol{\beta}}_{k}$, and $\hat{\mathbf{D}}_{k}=\operatorname{diag}\left(\hat{\sigma}_{1 k}^{2} I, \hat{\sigma}_{2 k}^{2} I\right)$ compose current parameter estimates. Taking the posterior expectation of $L_{c}$ gives

$$
\mathbf{Q}=E\left\{L_{c} \mid \mathbf{Y}, \mathbf{T}, \boldsymbol{\Theta}\right\}=\sum_{i=1}^{n} \sum_{k=1}^{K} \omega_{i k} \log \tau_{k} \phi\left(\mathbf{y}_{i} \mid \mathbf{T}_{i} \boldsymbol{\beta}_{k}, \mathbf{D}_{k}\right) .
$$


Hence, the parameter estimates updated iteratively in the $\mathbf{M}$ step are obtained by maximizing the quantity $\mathbf{Q}$ with respect to $\left\{\tau_{k}, \beta_{1 k}, \beta_{2 k}, \sigma_{1 k}^{2}, \sigma_{2 k}^{2}\right\}$, which yields

$$
\begin{aligned}
\hat{\boldsymbol{\beta}}_{s k} & =\left[\hat{\omega}_{i, k} \mathbf{X}_{i}^{\mathrm{T}} \mathbf{X}_{i}\right]^{-1} \sum_{i=1}^{n} \hat{\omega}_{i, k} \mathbf{X}_{i}^{\mathrm{T}} \mathbf{y}_{s i}, \\
\hat{\boldsymbol{\sigma}}_{s k}^{2} & =\frac{1}{\sum_{i=1}^{n} \hat{\omega}_{i, k}} \sum_{i=1}^{n} \hat{\omega}_{i, k} \mathbf{y}_{s i}-\mathbf{X}_{i} \boldsymbol{\beta}_{s k}^{2} \text { for } s=1,2, \text { and } \\
\hat{\tau}_{k} & =\frac{1}{n} \sum_{i=1}^{n} \hat{\omega}_{i, k},
\end{aligned}
$$

where $\hat{\omega}_{i, k}$ are current estimates of the posterior probabilities. At the end of this process, cluster assignments are performed based on maximal posterior probabilities; that is, for each $i$

$$
\hat{z}_{i}=G \quad \text { such that } \quad \hat{\omega}_{i G}^{(\text {end) }}=\underset{k}{\operatorname{argmax}} \hat{\omega}_{i k}^{(\text {end) }} .
$$

Note that on our data the individual forecast tracks $\mathbf{y}_{i}$ cover $120 \mathrm{~h}$ (5 days) at 6-h intervals, so $\max \left(n_{i}\right)=21$ and the $t_{i}$ s are $0,6,12, \ldots \mathrm{h}$, up to a maximum of $120 \mathrm{~h}$.

The iterative EM algorithm needs to be initialized. This can be done in several ways; we initialize the $M$ step with random values for the $\hat{\omega}_{i, k}$ s (see also Gaffney 2004). To increase the chances of reaching a global maximum of the log-likelihood, we use several random EM starts (e.g., 15) and take estimates from the one with the highest loglikelihood value.

Employing well-established curve clustering software (MATLAB toolbox CCToolBox) on the 50-member forecast corresponding to a fixed initialization time and storm, and using 15 starts each time the EM is run, we compute traditional diagnostics for all 30 combinations of polynomial order and number of clusters in approximately $90-105$ s on a standard desktop computer. Calculation of cross-validation diagnostics can take as long as $130-140 \mathrm{~min}$. However, computation time decreases linearly with the number of EM starts; using 5 instead of 15 , cross-validation diagnostics could be computed for all specifications in $40-50 \mathrm{~min}$. In future applications, this time could be reduced to approximately $30 \mathrm{~min}$ by ignoring specifications that were found completely unsatisfactory in our study (e.g., first-order polynomials and/or two clusters).

\section{REFERENCES}

Arnott, J. M., J. L. Evans, and F. Chiaromonte, 2004: Characterization of extratropical transition using cluster analysis. Mon. Wea. Rev., 132, 2916-2937, doi:10.1175/MWR2836.1.

Barkmeijer, J., R. Buizza, T. Palmer, K. Puri, and J.-F. Mahouf, 2001: Tropical singular vectors computed with linearized diabatic physics. Quart. J. Roy. Meteor. Soc., 127, 685-708, doi:10.1002/qj.49712757221.

Berg, R., 2009: Tropical Cyclone Report Hurricane Ike. Rep. AL092008, National Hurricane Center, 55 pp.

Buizza, R., 2006: The ECMWF ensemble prediction system. Predictability of Weather and Climate, T. Palmer and R. Hagedorn, Eds., Cambridge University Press, 455-488.

- D. Richardson, and T. Palmer, 2003: Benefits of increased resolution in the ECMWF ensemble system and comparison with poor-man's ensembles. Quart. J. Roy. Meteor. Soc., 129, 1269-1288, doi:10.1256/qj.02.92.

Camargo, S. J., A. W. Robertson, S. J. Gaffney, P. Smyth, and M. Ghil, 2007: Cluster analysis of typhoon tracks. Part I: General properties. J. Climate, 20, 3635-3653, doi:10.1175/JCLI4188.1.

ECMWF, 2015: Operational configurations of the ECMWF Integrated Forecasting System (IFS). European Centre for Medium-Range Weather Forecasting, accessed 23 October 2015. [Available online at http://www.ecmwf.int/en/forecasts/ documentation-and-support.]

Evans, J. L., and R. E. Hart, 2003: Objective indicators of the life cycle evolution of extratropical transition for Atlantic tropical cyclones. Mon. Wea. Rev., 131, 909-925, doi:10.1175/ 1520-0493(2003)131<0909:OIOTLC>2.0.CO;2.

_ J. M. Arnott, and F. Chiaromonte, 2006: Evaluation of operational model cyclone structure forecasts during extratropical transition. Mon. Wea. Rev., 134, 3054-3072, doi:10.1175/ MWR3236.1.

Ferstl, F., K. Burger, and R. Westermann, 2016: Streamline variability plots for characterizing the uncertainty in vector field ensembles. IEEE Trans. Vis. Comput. Graph., 22, 767-776, doi:10.1109/TVCG.2015.2467204.

Fraley, C., and A. Raftery, 2009: MCLUST version 3 for R: Normal mixture modeling and model-based clustering. Tech. Rep. 504, University of Washington, Seattle, WA, 57 pp. [Available online at https://www.stat.washington.edu/research/reports/2006/tr504. pdf.]

Gaffney, S. J., 2004: Probabilistic curve-aligned clustering and prediction with regression mixture models. Ph.D. thesis, University of California, Irvine, CA, 281 pp. [Available online at http://www.ics. uci.edu/pub/sgaffney/outgoing/sgaffney_thesis.pdf.]

_ A. W. Robinson, P. Smith, S. J. Camargo, and M. Ghil, 2007: Probabilistic clustering of extratropical cyclones using regression mixture models. Climate Dyn., 29, 423-440, doi:10.1007/ s00382-007-0235-z.

Harr, P. A., and J. M. Dea, 2009: Downstream development associated with the extratropical transition of tropical cyclones over the western North Pacific. Mon. Wea. Rev., 137, 12951319, doi:10.1175/2008MWR2558.1.

— D. Anwender, and S. C. Jones, 2008: Predictability associated with the downstream impacts of the extratropical transition of tropical cyclones: Methodology and a case study of Typhoon Nabi (2005). Mon. Wea. Rev., 136, 3205-3225, doi:10.1175/ 2008MWR2248.1.

Hart, R. E., 2003: A cyclone phase space derived from thermal wind and thermal asymmetry. Mon. Wea. Rev., 131, 585-616, doi:10.1175/1520-0493(2003)131<0585:ACPSDF>2.0.CO;2.

_ J. L. Evans, and C. Evans, 2006: Synoptic composites of the extratropical transition life cycle of North Atlantic tropical cyclones: Factors determining posttransition evolution. Mon. Wea. Rev., 134, 553-578, doi:10.1175/MWR3082.1.

Ieva, F., and A. M. Paganoni, 2013: Depth measures for multivariate functional data. Commun. Stat. Theory Methods, 42, 12651276, doi:10.1080/03610926.2012.746368. 
Jones, S. C., and Coauthors, 2003: The extratropical transition of tropical cyclones: Forecast challenges, current understanding, and future directions. Wea. Forecasting, 18, 1052-1092, doi:10.1175/ 1520-0434(2003)018<1052:TETOTC $>2.0$. CO;2.

JTWC, 2015: JTWC western North Pacific best track data. Joint Typhoon Warning Center, accessed 22 October 2015. [Available online at http://www.usno.navy.mil/NOOC/nmfc-ph/RSS/ jtwc/best_tracks/wpindex.php.]

Keller, J. H., S. C. Jones, J. L. Evans, and P. A. Harr, 2011: Characteristics of the TIGGE multimodel ensemble prediction system in representing forecast variability associated with extratropical transition. Geophys. Res. Lett., 38, L12802, doi:10.1029/2011GL047275.

- ——, and P. A. Harr, 2014: An eddy kinetic energy view of physical and dynamical processes in distinct forecast scenarios for the extratropical transition of two tropical cyclones. Mon. Wea. Rev., 142, 2751-2771, doi:10.1175/ MWR-D-13-00219.1.

Kozar, M. E., M. E. Mann, S. J. Camargo, J. P. Kossin, and J. L. Evans, 2012: Stratified statistical models of North Atlantic basin-wide and regional tropical cyclone counts. J. Geophys. Res., 117, D18103, doi:10.1029/2011JD017170.

Leutbecher, M., and T. N. Palmer, 2008: Ensemble forecasting. J. Comput. Phys., 227, 3515-3539, doi:10.1016/ j.jcp.2007.02.014.

López-Pintado, S., and R. Jornsten, 2007: Functional analysis via extensions of the band depth. Lect. Notes Monogr. Ser., 54, 103-120, doi:10.1214/074921707000000085.
_ - Y. Sun, J. K. Lin, and M. G. Genton, 2014: Simplicial band depth for multivariate functional data. Adv. Data Anal. Classif., 8, 321-338, doi:10.1007/s11634-014-0166-6.

McLachlan, G. J., and K. E. Basford, 1987: Mixture Models: Inference and Applications to Clustering. CRC Press, 272 pp.

Mirzargar, M., R. T. Whitaker, and R. M. Kirby, 2014: Curve boxplot: Generalization of boxplot for ensembles of curves. IEEE Trans. Visualization Comput. Graphics, 20, 2654-2663, doi:10.1109/TVCG.2014.2346455.

NHC, 2015: National Hurricane Center Forecast Verification. National Hurricane Center, accessed 23 October 2015. [Available online at http://www.nhc.noaa.gov/verification/ verify5.shtml.]

Paliwal, M., and A. Patwardhan, 2013: Identification of clusters in tropical cyclone tracks of North Indian Ocean. Nat. Hazards, 68, 645-656, doi:10.1007/s11069-013-0641-y.

Puri, K., J. Barkmeijer, and T. Palmer, 2001: Ensemble prediction of tropical cyclones using targeted diabatic singular vectors. Quart. J. Roy. Meteor. Soc., 127, 709-731, doi:10.1002/qj.49712757222.

Veren, D., J. L. Evans, S. Jones, and F. Chiaromonte, 2009: Novel metrics for evaluation of ensemble forecasts of tropical cyclone structure. Mon. Wea. Rev., 137, 2830-2850, doi:10.1175/ 2009MWR2655.1.

Whitaker, R. T., M. Mirzargar, and R. M. Kirby, 2013: Contour boxplots: A method for characterizing uncertainty in feature sets from simulation ensembles. IEEE Trans. Visualization Comput. Graphics, 19, 2713-2722, doi:10.1109/ TVCG.2013.143. 